\title{
STUDIES IN RENAL CIRCULATION DURING PERIODS OF LIFE STRESS AND ACCOMPANYING EMOTIONAL REACTIONS IN SUBJECTS WITH AND WITHOUT ESSENTIAL HYPERTENSION; OBSERVATIONS ON THE ROLE OF NEURAL ACTIVITY IN REGULATION OF RENAL BLOOD FLOW ${ }^{1}$
}

\author{
By JOHN B. PFEIFFER, JR., AND HAROLD G. WOLFF WITH THE teChNICAL \\ ASSISTANCE OF O. S. WINTER \\ (From the New York Hospital and the Departments of Medicine (Neurology) and Psychiatry \\ of Cornell University Medical College, New York, N. Y.)
}

(Submitted for publication July 28, 1949; accepted, June 5, 1950)

\section{INTRODUCTION}

The development of relatively simple methods for measuring renal blood flow and glomerular filtration rate by Smith and associates (2) has made it possible to investigate with safety variations in renal hemodynamics and function in the human subject. The renal blood flow has been found to be relatively stable in the standard ${ }^{2}$ state but it can be modified by various drugs and during emotional and physical stress. Head pain and "cold" pain $(3,4)$ produce a decrease in renal blood flow with relatively little change in glomerular filtration rate, similar to the effect produced by epinephrin and the more powerful sympathomimetic drugs (5). This same pattern of response accompanied reactions to stress which occurred fortuitously when two human subjects were under study by Homer Smith (6) : in one, when events were misinterpreted as threats of early and undeserved discharge from the hospital, and in the other, when the significance of the clearance procedures was interpreted by the subject as being ominous. In one of these patients arterial pressure was found to be slightly elevated during the

\footnotetext{
1 A preliminary report of some of these observations has already been made ( 1 ). This communication is a more complete statement of the experimental data and inferences concerning renal circulation. Special appreciation and acknowledgement are due Drs. Stewart Wolf and Herbert S. Ripley who are entirely responsible for the creation of the situations and feeling states that were necessary to the conduct of these experiments. A fuller statement of the types of conflict induced by interviews in these studies as well as the personality factors and reaction patterns pertinent to the problem of essential hypertension will be published at a later date.

2 The term "standard" is used because it is doubtful that under the conditions of the experimental procedure, the patient's condition can be truly "basal."
}

stress so that the results were not due to renal vasoconstriction or renal ischemia associated with shock and fall in cardiac output.

In this study, variations in renal hemodynamics and arterial blood pressure were investigated before, during, and after periods of emotional conflict induced by discussion of topics of important personal significance. States of overwhelming fear were avoided. In this manner, it was hoped that bodily reactions of the subject in his average daily experience would be revealed. It has been suggested that the kidney is implicated in the genesis and perpetuation of the elevated blood pressure in essential hypertension (7) and it has also been repeatedly demonstrated that the level of blood pressure varies widely with changing stress in the life situation (8-11). Therefore, renal hemodynamics and blood pressure variations were investigated to ascertain whether there was a consistent pattern or any significant difference in the degree of response in the hypertensive as compared to the normotensive subject during periods of experimentally induced conflict.

\section{METHOD}

Thirty-seven subjects were selected for study from patients at the New York Hospital. Their ages varied from 23 to 57 years. Emotional conflict was induced in 35 subjects, 14 male and 21 female. One subject was studied during relaxation only and another subject with a unilateral sympathectomy was considered not to have responded to the methods used to induce emotional conflict. The 13 subjects with average control blood pressures below 140/90 were arbitrarily classified as normotensives and the remaining 23 with average control blood pressures above this level, as hypertensives. All patients were free from renal diseases at least as far as clinical course, complete urine examinations, blood urea nitrogen determinations, phenolsulphonthalein excretion and urine concentration tests could be used as evidence. 
Those with arterial hypertension had had intensive personality studies. The normotensive subjects had been admitted to the hospital for complaints based on some structural disorder such as herniated nucleus pulposis or nerve injury, considered to have per se no significant effect on renal function and hemodynamics. Six of the hypertensive group were studied before and after lumbodorsal sympathectomies and splanchnicectomies as described by Smithwick (12). The sympathetic ganglia and chain were removed bilaterally from $T-7$ or $T-8$ to L-2 inclusive, together with section of the greater splanchnic nerves bilaterally in a two stage procedure. ${ }^{8}$

The experimental procedure for studying renal hemodynamics as described by Goldring and Chasis (2, Appendix E. pp 212-215) was identical for both the normotensive and hypertensive subjects. One liter of water was imbibed between 10:00 p.m. and 12:00 midnight the evening preceding, and again another liter between 7:00 a.m. and 7:30 a.m. the morning of the experiment. Breakfast was omitted. All the subjects were catheterized with a No. 16 French soft rubber catheter which had been modified by cutting several additional eyelets along the distal $3 \mathrm{~cm}$. After the catheter had been shown to drain the bladder completely, the control specimens of urine and blood were collected. After intravenous administration of the "priming" solution of sodium paraaminohippurate and mannitol, infusion of these same chemicals was continued for the duration of the experiment at the rate of $4 \mathrm{cc}$. per minute. Forty to $45 \mathrm{~min}$ utes were permitted to elapse to allow stabilization of urine flow and blood levels of the chemicals utilized, before the first test specimens were collected. Blood specimens were collected every 30 to 40 minutes thereafter.

The periods of clearance measurement were from 15 to 20 minutes each in duration with collection of urine samples at the end of each period; the average experiment was from four to four and one-half hours in duration of which two to two and one-half hours were devoted to clearance measurements. At the termination of each period, the bladder was rinsed with $50 \mathrm{cc}$. of sterile saline followed by $50 \mathrm{cc}$. of air. The first two to three periods were control periods. During the whole manipulation, an effort was made to induce a feeling of trust and relaxation in the subject, by engaging him in reassuring and neutral conversation. Only one observer was with the subject during the entire procedure except during the interview, when topics known to be of personal significance to him were discussed by a second experimenter. The interview was continued for 30 to 40 minutes during which one or two clearance measurements were made. Thereafter, the subject was again reassured and urged to relax. The clearance determinations were continued for another 45 to 60 minutes. The blood pressure was measured at five minute intervals during the control and post-discussion

8 These operations were performed by Drs. Bronson Ray, Charles Neill and Arthur Console of the Department of Surgery of the New York Hospital. It was through their cooperation and courtesy that we were able to study these patients. periods and at 30 second to two minute intervals throughout the discussion. It is not to be inferred that the subjects were ever completely relaxed but merely that they were more relaxed at certain times, that is, during the control and post-discussion periods, than during the discussion period.

Sodium para-aminohippurate (PAH) was used for the determination of effective renal plasma flow and mannitol (in a few instances inulin) for glomerular filtration rate. The methods of chemical determination were as follows: the PAH by the methods of Goldring and Chasis (2, Appendix B, p. 198 and Appendix D, pp. 203-204), inulin by a modification (13) of the method of Hubbard and Loomis (14) and mannitol by the method of Corcoran and Page (15). Commercial heparin solution was used as the anticoagulant. Hematocrit determinations were made on the control and final blood specimens. These were averaged for calculation of renal blood flow, but rarely was the variation between the two greater than 0.5 volume $\%$.

All clearances were corrected for surface area using the formula, 4

$$
\text { (1) } \mathrm{EPF}_{\text {corr. }}=\mathrm{EPF}_{\text {obs. }} \times \frac{1.73 \mathrm{sq} . \mathrm{m} .}{\text { surface area }} \text {. }
$$

Effective blood flow was calculated by the formula,

$$
\text { (2) } \mathrm{EBF}=\frac{\mathrm{EPF}}{1-\text { hematocrit }} \text {. }
$$

Mean blood pressure was calculated from the sphygmomanometer readings of systolic and diastolic blood pressures. In order to minimize the effect of sharply peaked pulse wave forms, $40 \%$ of the pulse pressure was added to the diastolic pressure,

$$
\text { (3) } \mathrm{MBP}=\mathrm{DBP}+0.4(\mathrm{SBP}-\mathrm{DBP}) \text {. }
$$

The resistance of the renal vascular system was approximated by using the ratio of the mean blood pressure to the effective blood flow,

(4) $\mathrm{PRU}=\frac{\mathrm{MBP}}{\mathrm{EBF}}$.

In the calculation of afferent and efferent arteriolar resistances, the formulae of Lamport (16) were used and

\footnotetext{
4 The abbreviations used in formulae (1) to (10) inclusive are as follows:

EPF-Effective plasma flow,

EBF-Effective blood flow,

SBP-Systolic blood pressure ( $\mathrm{mm} . \mathrm{Hg}$ ),

DBP_Diastolic blood pressure $(\mathrm{mm} . \mathrm{Hg})$,

MBP-Mean blood pressure ( $\mathrm{mm}$. $\mathrm{Hg}$ ),

PRU-Peripheral resistance units ( $\mathrm{mm} . \mathrm{Hg} / \mathrm{cc}$./min.),

$\mathrm{R}_{\mathrm{z}}-$ Afferent arteriolar resistance $(\mathrm{mm}$. $\mathrm{Hg} / \mathrm{cc} . / \mathrm{min}$.),

$\mathrm{R}_{\mathrm{P}}$-Efferent arteriolar resistance ( $\mathrm{mm} . \mathrm{Hg} / \mathrm{cc} . / \mathrm{min}$.),

$\mathbf{P}_{\mathbf{m}}$-Calculated mean blood pressure,

Po-Calculated osmotic pressure,

FF-Filtration fraction,

Co-Hematocrit,

$E_{z}$ and $E_{r}-$ Permeability Coefficients of the glomeruli and tubules, respectively.
} 
for comparison, the formulae of Gomez $(17,18)$ were used for the efferent arteriolar resistances only. The formulae of Gomez for afferent arteriolar resistance could not be used as values for certain constants were unobtainable and are so placed in the equations that percentage changes could not be calculated. The following formulae of Lamport were used:
(5) $\mathrm{R}_{\mathrm{a}}=\frac{\mathrm{P}_{\mathrm{m}}-\mathrm{P}_{0}-40}{\mathrm{EBF}}$,
(б) $R_{0}=\frac{\left(P_{0}-16.4\right)(1-0.47 \mathrm{FF})}{\mathrm{EBF}}$,
(7) $P_{m}=\frac{S B P+D B P}{2}$,
(8) $\mathrm{P}_{0}=\frac{16.38}{0.641-\mathrm{FF}}$.

The formulae of Gomez were rearranged and the following were derived:
(9) $R_{0}=\frac{F F\left(1-C_{0}\right)}{1-F F\left(1-C_{0}\right)}$,
(10) $\mathrm{K}=\frac{1}{\mathrm{E}_{\mathrm{g}}}+\frac{1}{\mathrm{E}_{\mathrm{r}}}$.

The constant " $K$ " cannot be evaluated as values for $E_{8}$ and $E_{r}$ cannot be obtained either from other sources or from manipulation of the equations with the data available. However, percentage changes can be calculated and used for comparison with percentage changes obtained by means of Lamport's formulae.

\section{A. Variations in blood pressure, renal blood flow, glomerular filtration rate and renal vas- cular resistance during emotional stress}

\section{Blood pressure}

The average blood pressures during the control periods of the experiment ranged from 95/58 to $228 / 148 \mathrm{~mm}$. $\mathrm{Hg}$ (Table I). Thirteen of the subjects considered normotensive exhibited average control blood pressures below 140/90 and calculated mean pressures below $110 \mathrm{~mm}$. $\mathrm{Hg}$. The average mean pressure in this group was 93.5 $\pm 9.9 \mathrm{~mm}$. $\mathrm{Hg}$ and for the hypertensive group was $137.3 \pm 19.4 \mathrm{~mm}$. Hg (Table II). During the discussion period, a rise was obtained in the majority of the subjects; although in some it was quite minor, the large number of blood pressures used to obtain the average figure given, makes even these small elevations significant. There was an average increase in mean blood pressure of 9.3 $\pm 5.7 \mathrm{~mm}$. in the normotensive group (10.1\% of mean pressure) and an increase of $17.3 \pm 10.8 \mathrm{~mm}$. in the hypertensive group (12.5\% of mean pres- sure). Although the changes produced in blood pressure during the discussion are highly significant, the difference in response between the two groups is barely so $(p=<0.02)$. When this change is considered as a percentage of the mean pressure, there is a trifling difference between the two groups.

Comment. It is evident that during certain types of emotional conflict, all of the subjects, hypertensives and normotensives alike, responded with increases in blood pressure, indicating that such a reaction is not peculiar to those with vascular hypertension but is part of a widely shared reaction to stress. There was no striking outward evidence that the subject was undergoing a strenuous emotional experience. However, it seems obvious that a similar percentage rise in blood pressure in normotensives and hypertensives would have more serious implications for the latter.

\section{Effective plasma flow, glomerular filtration rate and renal vascular resistance}

The average effective plasma flow when corrected for body surface area, was $509 \pm 94.2 \mathrm{cc}$./ min. This is low when compared to the figures given by Goldring and Chasis (2, p. 56), $697 \pm$ $151 \mathrm{cc} . / \mathrm{min}$. for males and $594 \pm 102 \mathrm{cc} . / \mathrm{min}$. for females. During the discussion periods, there was an average decrease of $-26.5 \pm 30.1 \mathrm{cc} . / \mathrm{min}$. in the effective plasma flow in the normotensive group. The hypertensive group had a still lower renal plasma flow $460 \pm 110 \mathrm{cc} . / \mathrm{min}$., and exhibited a decrease of $-42.4 \pm 52.6 \mathrm{cc}$. $/ \mathrm{min}$. during the period of stress. Although these changes are highly significant in each group, respectively, demonstrating the variability of renal blood flow with emotional stress, the intergroup difference is not significant (Table II). The glomerular filtration rates for the two groups were $89.1 \pm$ $11.9 \mathrm{cc} . / \mathrm{min}$. for the normotensives and $102.5 \pm$ $23.4 \mathrm{cc}$. $/ \mathrm{min}$. for the hypertensives. No significant changes occurred throughout the whole of the experiment in these values. In the filtration fraction, however, there were changes, and the deviation from the control values is significant. Intergroup differences (between normotensives and hypertensives), however, statistically are slight $(\mathrm{p}=<0.05)$ although there is an indication 
TABLE I

Summary of blood pressures, clearances and vascular resistances before, during and after interview*

\begin{tabular}{|c|c|c|c|c|c|c|c|c|c|c|c|c|c|}
\hline No. & Pt. & Sex & Proc. & B.P. & EPF & GFR & FF & $\stackrel{\Delta \%}{\mathrm{EPF}}$ & $\stackrel{\Delta \%}{\mathrm{GFR}}$ & $\stackrel{\Delta \%}{\mathrm{FF}}$ & EBF & PRU & $\stackrel{\Delta \%}{\text { PRU }}$ \\
\hline 1 & A. C. & F & $\begin{array}{r}\text { C } \\
\text { D } \\
P D\end{array}$ & $\begin{array}{l}95 / 58 \\
98 / 58 \\
93 / 52\end{array}$ & $\begin{array}{l}648 \\
658 \\
644\end{array}$ & $\begin{array}{l}97 \\
99 \\
89\end{array}$ & & $\begin{array}{l} \pm 0.7 \\
\pm \quad 1.5 \\
-0.6\end{array}$ & $\begin{array}{l} \pm 2.9 \\
+2.5 \\
-8.3\end{array}$ & $\begin{array}{l} \pm 3.3 \\
\pm 0.7 \\
-8.0\end{array}$ & $\begin{array}{r}998 \\
1011 \\
990\end{array}$ & & $\begin{array}{l} \pm 7.6 \\
\pm 0.0 \\
-1.4\end{array}$ \\
\hline 2 & A. $S$. & $\mathbf{M}$ & $\begin{array}{r}\mathrm{C} \\
\mathrm{D} \\
\mathrm{PD}\end{array}$ & $\begin{array}{l}107 / 69 \\
109 / 75 \\
114 / 78\end{array}$ & $\begin{array}{l}395 \\
363 \\
384\end{array}$ & $\begin{array}{l}75 \\
74 \\
81\end{array}$ & & $\begin{array}{l} \pm 2.5 \\
-8.1 \\
-2.8\end{array}$ & $\begin{array}{l} \pm 1.3 \\
=1.8 \\
+7.9\end{array}$ & $\begin{array}{r} \pm 2.6 \\
+\quad 6.8 \\
+8.4\end{array}$ & $\begin{array}{l}745 \\
685 \\
725\end{array}$ & $\begin{array}{l}0.133 \\
0.130 \\
0.127\end{array}$ & $\begin{array}{l} \pm 2.6 \\
+15.0 \\
+12.4\end{array}$ \\
\hline 3 & C. W. & F & $\begin{array}{r}\mathrm{C} \\
\mathrm{D} \\
\mathrm{PD}\end{array}$ & $\begin{array}{l}108 / 73 \\
112 / 79 \\
119 / 76\end{array}$ & $\begin{array}{l}439 \\
456 \\
439\end{array}$ & $\begin{array}{l}89 \\
90 \\
80\end{array}$ & $\begin{array}{l}0.203 \\
0.196 \\
0.183\end{array}$ & $\begin{array}{l} \pm 0.9 \\
+\quad 3.9 \\
\pm 0.0\end{array}$ & $\begin{array}{l} \pm 1.4 \\
+1.1 \\
-9.5\end{array}$ & $\begin{array}{l} \pm 1.5 \\
=3.0 \\
-9.4\end{array}$ & $\begin{array}{l}770 \\
800 \\
770\end{array}$ & $\begin{array}{l}0.113 \\
0.115 \\
0.121\end{array}$ & $\begin{array}{l} \pm 1.8 \\
+1.8 \\
+7.1\end{array}$ \\
\hline 4 & J. L. & F & $\begin{array}{r}\text { C } \\
\text { D } \\
P D\end{array}$ & $\begin{array}{l}115 / 69 \\
122 / 79 \\
111 / 69\end{array}$ & $\begin{array}{l}636 \\
575 \\
594\end{array}$ & $\begin{array}{l}107 \\
107 \\
105\end{array}$ & $\begin{array}{l}0.168 \\
0.186 \\
0.177\end{array}$ & $\begin{array}{l} \pm 1.7 \\
=9.6 \\
-6.3\end{array}$ & $\begin{array}{l} \pm 6.7 \\
\pm 0.0 \\
-1.8\end{array}$ & $\begin{array}{r}+5.9 \\
+13.1 \\
+\quad 5.4\end{array}$ & $\begin{array}{l}1179 \\
1065 \\
1100\end{array}$ & $\begin{array}{l}0.074 \\
0.090 \\
0.078\end{array}$ & $\begin{array}{l} \pm 2.6 \\
+21.6 \\
+5.4\end{array}$ \\
\hline 5 & J. S. & F & $\begin{array}{r}\text { C } \\
\text { D } \\
P D\end{array}$ & $\begin{array}{l}114 / 70 \\
122 / 77 \\
115 / 71\end{array}$ & $\begin{array}{l}469 \\
444 \\
494\end{array}$ & $\begin{array}{l}92 \\
93 \\
90\end{array}$ & $\begin{array}{l}0.196 \\
0.208 \\
0.182\end{array}$ & $\begin{array}{l} \pm 5.8 \\
\pm 5.3 \\
+5.3\end{array}$ & $\begin{array}{l} \pm 2.4 \\
+1.1 \\
-2.2\end{array}$ & $\begin{array}{l} \pm 3.1 \\
+6.1 \\
-7.7\end{array}$ & $\begin{array}{l}740 \\
700 \\
778\end{array}$ & & $\begin{array}{r} \pm 5.5 \\
+14.3 \\
-4.1\end{array}$ \\
\hline 6 & C. S. & F & $\begin{array}{r}\mathrm{C} \\
\mathrm{D} \\
\mathrm{PD}\end{array}$ & $\begin{array}{l}118 / 72 \\
154 / 89 \\
141 / 84\end{array}$ & $\begin{array}{l}583 \\
496 \\
514\end{array}$ & $\begin{array}{l}77 \\
74 \\
80\end{array}$ & $\begin{array}{l}0.132 \\
0.148 \\
0.151\end{array}$ & $\begin{array}{l} \pm 0.7 \\
-14.9 \\
-11.8\end{array}$ & $\begin{array}{l} \pm 1.3 \\
-4.0 \\
+4.8\end{array}$ & $\begin{array}{r} \pm 0.8 \\
+12.1 \\
+18.2\end{array}$ & $\begin{array}{r}1040 \\
886 \\
917\end{array}$ & & $\begin{array}{l} \pm 3.4 \\
+49.5 \\
+34.5\end{array}$ \\
\hline 7 & J. K. & F & $\begin{array}{r}\mathrm{C} \\
\mathrm{D} \\
\mathrm{PD}\end{array}$ & $\begin{array}{l}125 / 78 \\
141 / 86 \\
126 / 79\end{array}$ & $\begin{array}{l}561 \\
520 \\
479\end{array}$ & $\begin{array}{l}81 \\
76 \\
70\end{array}$ & & $\begin{array}{l} \pm 7.6 \\
=7.1 \\
-14.6\end{array}$ & $\begin{array}{l} \pm 5.6 \\
=6.2 \\
-13.6\end{array}$ & $\begin{array}{l} \pm 1.4 \\
+1.4 \\
+1.4\end{array}$ & $\begin{array}{l}935 \\
867 \\
782\end{array}$ & & $\begin{array}{r} \pm 5.3 \\
+20.2 \\
+20.2\end{array}$ \\
\hline 8 & R.S. & F & $\begin{array}{r}\mathrm{C} \\
\mathrm{D} \\
\mathrm{PD}\end{array}$ & & $\begin{array}{l}361 \\
368 \\
353\end{array}$ & $\begin{array}{l}68 \\
70 \\
69\end{array}$ & & $\begin{array}{l} \pm 4.5 \\
\pm \quad 1.2 \\
-2.2\end{array}$ & $\begin{array}{l}+4.5 \\
+\quad 2.9 \\
+\quad 1.4\end{array}$ & $\begin{array}{l} \pm 0.5 \\
+\quad 1.1 \\
+4.2\end{array}$ & $\begin{array}{l}694 \\
708 \\
679\end{array}$ & & $\begin{array}{l} \pm 4.7 \\
+7.1 \\
+3.6\end{array}$ \\
\hline 9 & B. $Z$. & F & $\begin{array}{r}\text { C } \\
\text { D } \\
P D\end{array}$ & & & $\begin{array}{l}77 \\
75 \\
78\end{array}$ & & $\begin{array}{l} \pm 0.5 \\
=2.5 \\
=1.8\end{array}$ & $\begin{array}{l} \pm 3.0 \\
-2.8 \\
+\quad 1.1\end{array}$ & $\begin{array}{l} \pm 3.6 \\
+\quad 0.5 \\
+\quad 2.3\end{array}$ & $\begin{array}{l}733 \\
715 \\
724\end{array}$ & & $\begin{array}{r}0.7 \\
+\quad 0.7 \\
+\quad 3.0\end{array}$ \\
\hline 10 & B. S. & $\mathbf{M}$ & $\begin{array}{r}\text { C } \\
\text { D } \\
P D\end{array}$ & $\begin{array}{l}130 / 79 \\
137 / 84 \\
129 / 78\end{array}$ & $\begin{array}{l}516 \\
472 \\
505\end{array}$ & $\begin{array}{l}98 \\
95 \\
97\end{array}$ & $\begin{array}{l}0.173 \\
0.201 \\
0.192\end{array}$ & $\begin{array}{l} \pm 4.0 \\
-8.5 \\
-2.3\end{array}$ & $\begin{array}{l} \pm 3.0 \\
=3.1 \\
=1.0\end{array}$ & $\begin{array}{r}+3.7 \\
+16.2 \\
+11.0\end{array}$ & $\begin{array}{l}958 \\
875 \\
935\end{array}$ & & $\begin{array}{l} \pm 4.7 \\
+16.5 \\
+\quad 1.9\end{array}$ \\
\hline 11 & M. A. & F & $\begin{array}{r}\text { C } \\
\text { D } \\
\text { PD } \\
P D\end{array}$ & $\begin{array}{l}134 / 82 \\
157 / 95 \\
146 / 85 \\
134 / 82\end{array}$ & $\begin{array}{l}556 \\
507 \\
571 \\
662\end{array}$ & $\begin{array}{l}106 \\
106 \\
113 \\
106\end{array}$ & & $\begin{array}{r} \pm 5.8 \\
\pm \quad 8.8 \\
+2.7 \\
+18.8\end{array}$ & $\begin{array}{l} \pm 5.7 \\
\pm 0.0 \\
+6.6 \\
\pm 0.0\end{array}$ & $\begin{array}{r} \pm 4.3 \\
+9.4 \\
+3.7 \\
-15.2\end{array}$ & $\begin{array}{r}1030 \\
940 \\
1058 \\
1224\end{array}$ & & $\begin{array}{r} \pm 4.9 \\
+28.0 \\
+3.0 \\
-16.0\end{array}$ \\
\hline 12 & M. B. & F & $\begin{array}{r}\text { C } \\
\text { D } \\
P D\end{array}$ & $\begin{array}{l}136 / 84 \\
139 / 87 \\
134 / 87\end{array}$ & $\begin{array}{l}540 \\
518 \\
531\end{array}$ & $\begin{array}{r}97 \\
98 \\
100\end{array}$ & $\begin{array}{l}0.180 \\
0.194 \\
0.188\end{array}$ & $\begin{array}{l} \pm 3.8 \\
\pm 4.1 \\
-1.7\end{array}$ & $\begin{array}{l} \pm 3.1 \\
+\quad 1.0 \\
+\quad 2.1\end{array}$ & $\begin{array}{l} \pm 2.8 \\
+\quad 5.0 \\
+4.7\end{array}$ & $\begin{array}{r}1000 \\
960 \\
984\end{array}$ & & $\begin{array}{l} \pm 4.7 \\
+7.6 \\
+2.9\end{array}$ \\
\hline 13 & C. $\mathrm{H}$. & $\mathbf{M}$ & $\begin{array}{r}\text { C } \\
\text { D } \\
P D\end{array}$ & & & $\begin{array}{l}94 \\
97 \\
96\end{array}$ & & $\begin{array}{l} \pm 2.7 \\
=1.4 \\
-0.8\end{array}$ & $\begin{array}{l} \pm 1.3 \\
+3.2 \\
+2.1\end{array}$ & $\begin{array}{r} \pm 4.0 \\
+\quad 4.4 \\
+\quad 2.7\end{array}$ & $\begin{array}{l}959 \\
945 \\
950\end{array}$ & & $\begin{array}{r} \pm 4.9 \\
+11.5 \\
+1.0\end{array}$ \\
\hline 14 & J. R. & $\mathbf{M}$ & $\begin{array}{r}\mathrm{C} \\
\mathrm{D} \\
\mathrm{PD}\end{array}$ & $\begin{array}{l}139 / 93 \\
149 / 96 \\
131 / 83\end{array}$ & $\begin{array}{l}599 \\
498 \\
496\end{array}$ & $\begin{array}{l}122 \\
119 \\
125\end{array}$ & $\begin{array}{l}0.204 \\
0.239 \\
0.252\end{array}$ & $\begin{array}{r} \pm 7.7 \\
-16.9 \\
-17.2\end{array}$ & $\begin{array}{l}+3.7 \\
\pm 2.5 \\
+2.5\end{array}$ & $\begin{array}{r} \pm 3.0 \\
+17.2 \\
+23.5\end{array}$ & $\begin{array}{r}1151 \\
957 \\
954\end{array}$ & & $\begin{array}{r} \pm 2.1 \\
+20.8 \\
+11.5\end{array}$ \\
\hline 15 & R. G. & $\mathbf{M}$ & $\begin{array}{r}\text { C } \\
\text { D } \\
P D\end{array}$ & $\begin{array}{l}159 / 81 \\
160 / 84 \\
160 / 83\end{array}$ & $\begin{array}{l}325 \\
300 \\
293\end{array}$ & $\begin{array}{l}80 \\
77 \\
79\end{array}$ & $\begin{array}{l}0.246 \\
0.253 \\
0.269\end{array}$ & $\begin{array}{l} \pm 5.5 \\
=7.7 \\
-9.8\end{array}$ & $\begin{array}{l} \pm 4.9 \\
=3.7 \\
=1.3\end{array}$ & $\begin{array}{r} \pm 1.0 \\
+\quad 2.9 \\
+9.3\end{array}$ & $\begin{array}{l}625 \\
576 \\
563\end{array}$ & & $\begin{array}{r} \pm 0.7 \\
+10.6 \\
+20.1\end{array}$ \\
\hline 16 & M. B. & $\mathbf{M}$ & $\begin{array}{r}\text { C } \\
\text { D } \\
P D \\
P D\end{array}$ & $\begin{array}{l}149 / 96 \\
185 / 114 \\
182 / 110 \\
168 / 112\end{array}$ & $\begin{array}{l}463 \\
441 \\
438 \\
467\end{array}$ & $\begin{array}{l}103 \\
220 \\
126 \\
113\end{array}$ & $\begin{array}{l}0.223 \\
0.272 \\
0.289 \\
0.247\end{array}$ & $\begin{array}{r} \pm 4.4 \\
\pm \quad 4.3 \\
=5.8 \\
+0.9\end{array}$ & $\begin{array}{r} \pm 0.7 \\
+16.5 \\
+22.3 \\
+9.7\end{array}$ & $\begin{array}{r} \pm 4.5 \\
+22.0 \\
+29.6 \\
+10.7\end{array}$ & $\begin{array}{l}857 \\
816 \\
887 \\
865\end{array}$ & $\begin{array}{l}0.136 \\
0.174 \\
0.172 \\
0.155\end{array}$ & $\begin{array}{r}+2.8 \\
+27.9 \\
+26.5 \\
+14.0\end{array}$ \\
\hline 18 & B. A. & $\mathbf{M}$ & $\begin{array}{r}\text { C } \\
\text { D } \\
\text { PD }\end{array}$ & $\begin{array}{l}154 / 94 \\
161 / 98 \\
140 / 88\end{array}$ & $\begin{array}{l}472 \\
374 \\
467\end{array}$ & $\begin{array}{l}81 \\
80 \\
85\end{array}$ & $\begin{array}{l}0.172 \\
0.214 \\
0.182\end{array}$ & $\begin{array}{l} \pm 5.3 \\
=21.6 \\
-\quad 1.1\end{array}$ & $\begin{array}{l} \pm 5.4 \\
\pm \quad 1.2 \\
+4.9\end{array}$ & $\begin{array}{r} \pm 3.5 \\
+24.4 \\
+\quad 5.8\end{array}$ & $\begin{array}{l}875 \\
692 \\
865\end{array}$ & $\begin{array}{l}0.135 \\
0.178 \\
0.126\end{array}$ & $\begin{array}{r} \pm 5.8 \\
+31.9 \\
-6.7\end{array}$ \\
\hline
\end{tabular}


TABLE I-Continued

\begin{tabular}{|c|c|c|c|c|c|c|c|c|c|c|c|c|c|}
\hline No. & Pt. & Sex & Proc. & B.P. & EPF & GFR & FF & EPF & $\Delta \%$ & $\stackrel{\Delta}{\mathrm{F}}$ & EBF & PRU & PRU \\
\hline 19 & S. G. & $\mathbf{M}$ & $\begin{array}{r}\mathrm{C} \\
\mathrm{D} \\
\mathrm{PD}\end{array}$ & $\begin{array}{l}142 / 102 \\
154 / 110 \\
148 / 104\end{array}$ & $\begin{array}{l}407 \\
321 \\
337\end{array}$ & $\begin{array}{r}100 \\
85 \\
82\end{array}$ & $\begin{array}{l}0.245 \\
0.265 \\
0.233\end{array}$ & $\begin{array}{l} \pm 2.0 \\
=21.2 \\
-17.2\end{array}$ & $\begin{array}{l} \pm 3.1 \\
-15.0 \\
-18.0\end{array}$ & $\begin{array}{l} \pm 2.2 \\
\pm \quad 6.7 \\
-5.4\end{array}$ & & & $\begin{array}{l} \pm 3.1 \\
+35.7 \\
+23.6\end{array}$ \\
\hline 22 & M. B. & F & $\begin{array}{r}\mathrm{C} \\
\mathrm{PD}\end{array}$ & $\begin{array}{l}165 / 106 \\
180 / 121 \\
166 / 106\end{array}$ & $\begin{array}{l}503 \\
528 \\
471\end{array}$ & $\begin{array}{l}108 \\
120 \\
106\end{array}$ & $\begin{array}{l}0.215 \\
0.228 \\
0.224\end{array}$ & $\begin{array}{l} \pm 2.0 \\
\pm \quad 5.0 \\
-6.4\end{array}$ & $\begin{array}{r} \pm 2.9 \\
+11.4 \\
-\quad 2.1\end{array}$ & $\begin{array}{l} \pm 2.7 \\
+6.0 \\
+4.2\end{array}$ & $\begin{array}{l}852 \\
852 \\
895\end{array}$ & & $\begin{array}{l} \pm 2.5 \\
+11.1 \\
-\quad 5.2\end{array}$ \\
\hline 23 & R. C. & $\mathbf{M}$ & $\begin{array}{r}\mathrm{C} \\
\mathrm{PD}\end{array}$ & $\begin{array}{l}157 / 112 \\
164 / 116 \\
173 / 116\end{array}$ & $\begin{array}{l}455 \\
400 \\
410\end{array}$ & $\begin{array}{r}104 \\
98 \\
98\end{array}$ & $\begin{array}{l}0.229 \\
0.245 \\
0.239\end{array}$ & $\begin{array}{l} \pm 5.6 \\
-12.4 \\
-10.1\end{array}$ & $\begin{array}{l} \pm 3.8 \\
=5.8 \\
-5.8\end{array}$ & $\begin{array}{l} \pm 3.5 \\
+7.0 \\
+4.4\end{array}$ & $\begin{array}{r}1022 \\
899 \\
922\end{array}$ & & $\begin{array}{r} \pm 4.0 \\
+18.1 \\
+18.9\end{array}$ \\
\hline 24 & C. $\mathrm{K}$. & F & $\begin{array}{r}\text { C } \\
\text { D } \\
\text { PD } \\
\text { PD }\end{array}$ & $\begin{array}{l}168 / 111 \\
198 / 128 \\
170 / 112 \\
168 / 112\end{array}$ & $\begin{array}{l}663 \\
652 \\
597 \\
604\end{array}$ & $\begin{array}{l}156 \\
157 \\
153 \\
155\end{array}$ & & $\begin{array}{l} \pm 3.9 \\
\pm \quad 1.4 \\
-10.0 \\
-8.9\end{array}$ & $\begin{array}{l} \pm 3.2 \\
\pm 0.6 \\
\pm \quad 1.9 \\
-0.6\end{array}$ & $\begin{array}{r}+4.7 \\
+2.1 \\
+8.9 \\
+8.9\end{array}$ & $\begin{array}{r}1105 \\
1088 \\
995 \\
1006\end{array}$ & & $\begin{array}{r}+4.0 \\
+18.2 \\
+12.4 \\
+4.1\end{array}$ \\
\hline 25 & M. H. & F & $\begin{array}{l}\text { C } \\
\text { D }\end{array}$ & & $\begin{array}{l}704 \\
524\end{array}$ & $\begin{array}{l}121 \\
106\end{array}$ & & $\begin{array}{l} \pm 3.3 \\
-25.1\end{array}$ & $\begin{array}{l} \pm 2.0 \\
-9.3\end{array}$ & $\begin{array}{r} \pm 0.6 \\
+17.0\end{array}$ & $\begin{array}{r}1173 \\
875\end{array}$ & & $\begin{array}{r}\mathbf{2} 2.5 \\
+59.5\end{array}$ \\
\hline 26 & M. 0. & F & $\begin{array}{r}\text { C } \\
\text { D } \\
P D\end{array}$ & & $\begin{array}{l}504 \\
463 \\
448\end{array}$ & $\begin{array}{l}142 \\
144 \\
127\end{array}$ & & $\begin{array}{l} \pm 2.5 \\
=5.8 \\
-9.1\end{array}$ & $\begin{array}{l} \pm 3.0 \\
+1.4 \\
-10.6\end{array}$ & $\begin{array}{l} \pm 1.4 \\
+11.4 \\
+2.2\end{array}$ & & & $\begin{array}{l} \pm 3.2 \\
+17.6 \\
+4.4\end{array}$ \\
\hline 28 & R. H. & $\mathbf{M}$ & $\begin{array}{r}\text { C } \\
\text { D } \\
\text { PD }\end{array}$ & & $\begin{array}{l}430 \\
468 \\
417\end{array}$ & $\begin{array}{l}119 \\
118 \\
122\end{array}$ & $\begin{array}{l}0.277 \\
0.252 \\
0.299\end{array}$ & $\begin{array}{l} \pm 2.3 \\
\pm \quad 8.8 \\
-3.0\end{array}$ & $\begin{array}{l} \pm 3.4 \\
=0.8 \\
+\quad 2.5\end{array}$ & $\begin{array}{l} \pm 1.1 \\
=9.0 \\
+8.0\end{array}$ & $\begin{array}{l}852 \\
927 \\
826\end{array}$ & & $\begin{array}{l} \pm 1.2 \\
+\quad 2.5 \\
+3.1\end{array}$ \\
\hline 29 & N. 0. & F & $\begin{array}{r}\text { C } \\
\text { D } \\
P D\end{array}$ & $\begin{array}{l}180 / 112 \\
210 / 126 \\
198 / 120\end{array}$ & $\begin{array}{l}302 \\
279 \\
289\end{array}$ & $\begin{array}{l}70 \\
72 \\
68\end{array}$ & $\begin{array}{l}0.232 \\
0.258 \\
0.235\end{array}$ & $\begin{array}{l} \pm 2.1 \\
=7.6 \\
=4.3\end{array}$ & $\begin{array}{l} \pm 4.7 \\
\pm \quad 2.9 \\
-\quad 2.9\end{array}$ & $\begin{array}{l} \pm 3.4 \\
+11.4 \\
+1.3\end{array}$ & $\begin{array}{l}570 \\
527 \\
545\end{array}$ & & $\begin{array}{l} \pm 6.5 \\
+23.2 \\
+13.5\end{array}$ \\
\hline 31 & R. S. & F & $\begin{array}{r}\mathrm{C} \\
\mathrm{D} \\
\mathrm{PD}\end{array}$ & $\begin{array}{l}193 / 115 \\
207 / 120 \\
191 / 115\end{array}$ & $\begin{array}{l}472 \\
484 \\
451\end{array}$ & $\begin{array}{l}134 \\
139 \\
136\end{array}$ & $\begin{array}{l}0.284 \\
0.288 \\
0.301\end{array}$ & $\begin{array}{l} \pm 3.0 \\
\pm 2.5 \\
-4.5\end{array}$ & $\begin{array}{l} \pm 0.0 \\
+3.7 \\
+1.5\end{array}$ & $\begin{array}{l} \pm 2.1 \\
+\quad 3.7 \\
+1.5\end{array}$ & & & $\begin{array}{l} \pm 4.6 \\
+3.5 \\
+3.5\end{array}$ \\
\hline 33 & J. H. & $\mathbf{M}$ & $\begin{array}{r}\text { C } \\
\text { D } \\
P D\end{array}$ & & $\begin{array}{l}393 \\
391 \\
389\end{array}$ & $\bar{z}$ & $\bar{z}$ & $\begin{array}{l} \pm 1.5 \\
=0.5 \\
-1.0\end{array}$ & = & $=$ & $\begin{array}{l}727 \\
724 \\
720\end{array}$ & & $\begin{array}{l} \pm 4.8 \\
\pm \quad 7.1 \\
-2.7\end{array}$ \\
\hline 34 & C. $\mathrm{M}$. & $\mathbf{M}$ & $\begin{array}{r}\text { C } \\
\text { D } \\
P D\end{array}$ & $\begin{array}{l}208 / 129 \\
229 / 137 \\
214 / 128\end{array}$ & $\begin{array}{l}489 \\
489 \\
485\end{array}$ & $\begin{array}{l}102 \\
107 \\
101\end{array}$ & & $\begin{array}{l} \pm 3.7 \\
\pm 0.0 \\
-1.0\end{array}$ & $\begin{array}{l} \pm 4.4 \\
\pm 5.6 \\
-1.0\end{array}$ & $\begin{array}{l} \pm 1.0 \\
+5.8 \\
-0.2\end{array}$ & & & $\begin{array}{r} \pm 2.8 \\
+7.8 \\
+1.6\end{array}$ \\
\hline 35 & J. T. & $\mathbf{M}$ & $\begin{array}{r}\mathrm{C} \\
\mathrm{D} \\
\mathrm{PD}\end{array}$ & $\begin{array}{l}228 / 148 \\
243 / 157 \\
213 / 139\end{array}$ & $\begin{array}{l}306 \\
268 \\
306\end{array}$ & $\begin{array}{l}84 \\
79 \\
79\end{array}$ & $\begin{array}{l}0.274 \\
0.295 \\
0.258\end{array}$ & $\begin{array}{l} \pm 2.7 \\
-12.4 \\
\pm 0.0\end{array}$ & $\begin{array}{l} \pm 2.4 \\
=5.9 \\
-5.9\end{array}$ & $\begin{array}{r} \pm 1.7 \\
+7.8 \\
-5.8\end{array}$ & $\begin{array}{l}\mathbf{5 7 7} \\
504 \\
577\end{array}$ & & $\begin{array}{r} \pm 2.1 \\
+21.9 \\
-9.3\end{array}$ \\
\hline 36 & H. F. & $\mathbf{M}$ & $\begin{array}{r}\mathrm{C} \\
\mathrm{D} \\
\mathrm{PD}\end{array}$ & $\begin{array}{l}197 / 109 \\
152 / 92 \\
179 / 105\end{array}$ & $\begin{array}{l}450 \\
557 \\
472\end{array}$ & $\begin{array}{l}101 \\
122 \\
106\end{array}$ & $\begin{array}{l}0.227 \\
0.219 \\
0.225\end{array}$ & $\begin{array}{l} \pm 3.5 \\
-23.8 \\
+4.9\end{array}$ & $\begin{array}{l} \pm 4.8 \\
-18.8 \\
+5.0\end{array}$ & $\begin{array}{l} \pm 1.3 \\
=3.5 \\
-0.9\end{array}$ & $\begin{array}{r}834 \\
1031 \\
874\end{array}$ & $\begin{array}{l}0.173 \\
0.102 \\
0.154\end{array}$ & $\begin{array}{l} \pm 3.9 \\
-41.0 \\
-11.0\end{array}$ \\
\hline \multirow[t]{3}{*}{37} & H. W. & $\mathbf{F}$ & C & $107 / 73$ & $\begin{array}{l}\text { R-201 } \\
\text { L-165 }\end{array}$ & $\begin{array}{l}41 \\
33\end{array}$ & $\begin{array}{l}0.203 \\
0.202\end{array}$ & $\begin{array}{l} \pm 0.5 \\
\pm 3.0\end{array}$ & $\begin{array}{l} \pm 2.4 \\
\pm 4.3\end{array}$ & $\begin{array}{l} \pm 2.0 \\
+5.4\end{array}$ & $\begin{array}{l}347 \\
284\end{array}$ & $\begin{array}{l}0.251 \\
0.306\end{array}$ & $\begin{array}{r} \pm 0.5 \\
+3.0\end{array}$ \\
\hline & & & D & $112 / 79$ & $\begin{array}{l}\text { R-214 } \\
\text { L-169 }\end{array}$ & $\begin{array}{l}43 \\
32\end{array}$ & $\begin{array}{l}0.200 \\
0.190\end{array}$ & $\begin{array}{r}+6.4 \\
+2.2\end{array}$ & $\begin{array}{l} \pm 4.9 \\
-3.0\end{array}$ & $\begin{array}{l}-1.5 \\
-5.9\end{array}$ & $\begin{array}{l}368 \\
292\end{array}$ & & $\begin{array}{l}0.4 \\
+\quad 2.7\end{array}$ \\
\hline & & & PD & $110 / 76$ & $\begin{array}{l}\text { R-209 } \\
\text { L-160 }\end{array}$ & $\begin{array}{l}39 \\
29\end{array}$ & $\begin{array}{l}0.187 \\
0.186\end{array}$ & $\begin{array}{r}+4.0 \\
-2.2\end{array}$ & $\begin{array}{r}-4.9 \\
-12.1\end{array}$ & $\begin{array}{l}-7.8 \\
=7.8\end{array}$ & $\begin{array}{l}361 \\
264\end{array}$ & $\begin{array}{l}0.249 \\
0.341\end{array}$ & $\begin{array}{r}-0.5 \\
+11.1\end{array}$ \\
\hline
\end{tabular}

Studies pre- and post- sympathectomy

\begin{tabular}{|c|c|c|c|c|c|c|c|c|c|c|c|c|c|}
\hline $\begin{array}{l}17 \\
\text { Pre }\end{array}$ & R. W. & $\mathbf{F}$ & $\begin{array}{r}\text { C } \\
\text { D } \\
\text { PD }\end{array}$ & $\begin{array}{l}155 / 85 \\
188 / 104 \\
145 / 93\end{array}$ & $\begin{array}{l}587 \\
478 \\
451\end{array}$ & $\begin{array}{l}113 \\
114 \\
115\end{array}$ & $\begin{array}{l}0.193 \\
0.239 \\
0.255\end{array}$ & $\begin{array}{l} \pm 0.9 \\
-18.6 \\
-23.2\end{array}$ & $\begin{array}{l} \pm 1.2 \\
+0.9 \\
+1.8\end{array}$ & $\begin{array}{r} \pm 0.1 \\
+22.8 \\
+25.4\end{array}$ & $\begin{array}{r}1089 \\
885 \\
835\end{array}$ & $\begin{array}{l}0.104 \\
0.156 \\
0.137\end{array}$ & $\begin{array}{r}+1.4 \\
+50.0 \\
+31.7\end{array}$ \\
\hline
\end{tabular}


TABLE I-Continued

\begin{tabular}{|c|c|c|c|c|c|c|c|c|c|c|c|c|c|}
\hline No. & Pt. & Sex & Proc. & B.P. & EPF & GFR & FF & $\Delta \%$ & $\stackrel{\Delta \%}{G F R}$ & $\frac{\Delta \%}{\mathrm{~F} F}$ & EBF & PRU & PRU \\
\hline Post & & & $\begin{array}{r}\text { C } \\
\text { D } \\
\text { PD } \\
\text { PD }\end{array}$ & $\begin{array}{l}162 / 92 \\
182 / 110 \\
159 / 96 \\
148 / 88\end{array}$ & $\begin{array}{l}486 \\
478 \\
501 \\
503\end{array}$ & $\begin{array}{r}118 \\
115 \\
105 \\
97\end{array}$ & $\begin{array}{l}0.242 \\
0.240 \\
0.196 \\
0.198\end{array}$ & $\begin{array}{l} \pm 2.9 \\
\pm 2.0 \\
+3.5 \\
+3.1\end{array}$ & $\begin{array}{l} \pm 0.0 \\
=11.0 \\
-18.5 \\
-17.8\end{array}$ & $\begin{array}{l} \pm 2.9 \\
=0.8 \\
-19.2 \\
-18.2\end{array}$ & $\begin{array}{l}868 \\
854 \\
895 \\
899\end{array}$ & $\begin{array}{l}0.138 \\
0.163 \\
0.137 \\
0.120\end{array}$ & $\begin{array}{r} \pm 5.5 \\
+18.1 \\
-0.7 \\
-15.0\end{array}$ \\
\hline $\begin{array}{c}20 \\
\text { Pre }\end{array}$ & H. G. & F & $\begin{array}{r}\text { C } \\
\text { D } \\
\text { PD }\end{array}$ & $\begin{array}{l}164 / 91 \\
220 / 120 \\
194 / 106\end{array}$ & $\begin{array}{l}527 \\
483 \\
491\end{array}$ & $\begin{array}{l}88 \\
99 \\
84\end{array}$ & $\begin{array}{l}0.167 \\
0.205 \\
0.171\end{array}$ & $\begin{array}{l} \pm 1.7 \\
=8.3 \\
-6.8\end{array}$ & $\begin{array}{l} \pm 5.6 \\
+12.5 \\
-4.4\end{array}$ & $\begin{array}{l} \pm 4.3 \\
+22.7 \\
+\quad 2.4\end{array}$ & $\begin{array}{l}879 \\
805 \\
819\end{array}$ & $\begin{array}{l}0.190 \\
0.230 \\
0.184\end{array}$ & $\begin{array}{l} \pm 2.1 \\
+21.1 \\
+3.2\end{array}$ \\
\hline Post & & & $\begin{array}{r}\text { C } \\
\text { D } \\
P D\end{array}$ & $\begin{array}{l}176 / 103 \\
211 / 116 \\
174 / 103\end{array}$ & $\begin{array}{l}279 \\
324 \\
330\end{array}$ & $\begin{array}{l}75 \\
77 \\
71\end{array}$ & $\begin{array}{l}0.269 \\
0.237 \\
0.214\end{array}$ & $\begin{array}{l} \pm 1.1 \\
+16.8 \\
+18.3\end{array}$ & $\begin{array}{l} \pm 0.7 \\
\pm 2.3 \\
-6.8\end{array}$ & $\begin{array}{l} \pm 1.8 \\
-11.1 \\
-20.4\end{array}$ & $\begin{array}{l}481 \\
559 \\
569\end{array}$ & & $\begin{array}{r} \pm 0.3 \\
+0.7 \\
-16.0\end{array}$ \\
\hline $\begin{array}{c}21 \\
\text { Pre }\end{array}$ & w. & F & $\begin{array}{r}\text { C } \\
\text { D } \\
P D \\
P D\end{array}$ & $\begin{array}{l}165 / 102 \\
185 / 113 \\
168 / 102 \\
161 / 101\end{array}$ & $\begin{array}{l}381 \\
351 \\
397 \\
417\end{array}$ & $\begin{array}{l}76 \\
72 \\
75 \\
76\end{array}$ & $\begin{array}{l}0.198 \\
0.205 \\
0.189 \\
0.183\end{array}$ & $\begin{array}{l} \pm 2.9 \\
+7.9 \\
+4.2 \\
+9.5\end{array}$ & $\begin{array}{l} \pm 2.8 \\
\pm 4.5 \\
=0.4 \\
\pm 0.0\end{array}$ & $\begin{array}{l} \pm 2.0 \\
\pm 3.8 \\
\pm 4.6 \\
-0.1\end{array}$ & $\begin{array}{l}669 \\
621 \\
696 \\
732\end{array}$ & & $\begin{array}{l} \pm 2.1 \\
+21.1 \\
-3.2 \\
-10.0\end{array}$ \\
\hline Post & & & $\begin{array}{r}\text { C } \\
\text { D } \\
\text { PD } \\
\text { PD }\end{array}$ & $\begin{array}{l}175 / 110 \\
189 / 121 \\
174 / 116 \\
175 / 109\end{array}$ & $\begin{array}{l}372 \\
383 \\
395 \\
419\end{array}$ & $\begin{array}{l}68 \\
76 \\
71 \\
70\end{array}$ & $\begin{array}{l}0.184 \\
0.196 \\
0.180 \\
0.167\end{array}$ & $\begin{array}{l} \pm 1.1 \\
+3.0 \\
+6.1 \\
+12.6\end{array}$ & $\begin{array}{l} \pm 2.3 \\
+10.5 \\
+3.7 \\
+2.3\end{array}$ & $\begin{array}{l} \pm 3.3 \\
\pm \quad 7.3 \\
=2.2 \\
-9.2\end{array}$ & & & $\begin{array}{l} \pm 1.3 \\
+5.7 \\
-3.5 \\
-10.0\end{array}$ \\
\hline$\underset{\text { Pre }}{27}$ & B. $\mathrm{R}$. & F & $\begin{array}{r}\text { C } \\
\text { D } \\
P D\end{array}$ & $\begin{array}{l}179 / 110 \\
190 / 118 \\
184 / 115\end{array}$ & $\begin{array}{l}511 \\
399 \\
430\end{array}$ & $\begin{array}{r}101 \\
89 \\
95\end{array}$ & $\begin{array}{l}0.198 \\
0.223 \\
0.221\end{array}$ & $\begin{array}{l} \pm 5.7 \\
-21.9 \\
-16.0\end{array}$ & $\begin{array}{l} \pm 5.1 \\
-12.5 \\
-6.8\end{array}$ & $\begin{array}{l} \pm 4.1 \\
+12.4 \\
+11.4\end{array}$ & & & $\begin{array}{r} \pm 7.6 \\
+47.5 \\
+23.4\end{array}$ \\
\hline Post & & & $\begin{array}{r}\mathrm{C} \\
\mathrm{D} \\
\mathrm{PD}\end{array}$ & $\begin{array}{l}129 / 88 \\
147 / 95 \\
134 / 90\end{array}$ & $\begin{array}{l}588 \\
535 \\
509\end{array}$ & $\begin{array}{r}105 \\
97 \\
90\end{array}$ & $\begin{array}{l}0.17 .9 \\
0.182 \\
0.178\end{array}$ & $\begin{array}{l} \pm 4.5 \\
-9.1 \\
-13.4\end{array}$ & $\begin{array}{l} \pm 5.3 \\
=7.7 \\
-14.3\end{array}$ & $\begin{array}{l} \pm 3.4 \\
\pm 1.7 \\
-0.9\end{array}$ & & & $\begin{array}{l} \pm 5.6 \\
+27.6 \\
+19.8\end{array}$ \\
\hline $\begin{array}{l}30 \\
\text { Pre }\end{array}$ & E. W. & F & $\begin{array}{r}\text { C } \\
\text { D } \\
P D \\
P D\end{array}$ & $\begin{array}{l}173 / 123 \\
236 / 153 \\
189 / 126 \\
173 / 120\end{array}$ & $\begin{array}{l}317 \\
292 \\
288 \\
285\end{array}$ & $\begin{array}{l}67 \\
74 \\
67 \\
57\end{array}$ & $\begin{array}{l}0.212 \\
0.254 \\
0.234 \\
0.199\end{array}$ & $\begin{array}{l} \pm 4.1 \\
=7.9 \\
-9.2 \\
-10.1\end{array}$ & $\begin{array}{l} \pm 5.4 \\
+10.7 \\
\pm 0.0 \\
-13.9\end{array}$ & $\begin{array}{l} \pm 2.4 \\
+19.8 \\
+10.4 \\
-6.1\end{array}$ & & & $\begin{array}{l}+1.2 \\
+41.9 \\
+23.2 \\
+9.7\end{array}$ \\
\hline Post & & & $\begin{array}{r}\text { C } \\
\text { D }\end{array}$ & $\begin{array}{l}201 / 126 \\
227 / 143 \\
193 / 128\end{array}$ & $\begin{array}{l}297 \\
326 \\
312\end{array}$ & $\begin{array}{l}59 \\
68 \\
63\end{array}$ & $\begin{array}{l}0.199 \\
0.210 \\
0.203\end{array}$ & $\begin{array}{r} \pm 3.4 \\
+10.3 \\
+4.9\end{array}$ & $\begin{array}{r} \pm 4.8 \\
+15.2 \\
+\quad 6.8\end{array}$ & $\begin{array}{l} \pm 4.0 \\
+5.3 \\
+2.0\end{array}$ & & & $\begin{array}{l} \pm 5.6 \\
\pm \quad 2.9 \\
-5.7\end{array}$ \\
\hline $\begin{array}{l}32 \\
\text { Pre }\end{array}$ & R. L. & $\mathbf{M}$ & $\begin{array}{r}\mathrm{C} \\
\mathrm{D} \\
\mathrm{PD}\end{array}$ & $\begin{array}{l}219 / 113 \\
235 / 126 \\
217 / 112\end{array}$ & $\begin{array}{l}320 \\
314 \\
352\end{array}$ & $\begin{array}{l}82 \\
85 \\
84\end{array}$ & $\begin{array}{l}0.256 \\
0.272 \\
0.240\end{array}$ & $\begin{array}{l} \pm 6.8 \\
\pm 1.8 \\
+9.8\end{array}$ & $\begin{array}{l} \pm 5.2 \\
+4.4 \\
+3.3\end{array}$ & $\begin{array}{l} \pm 4.6 \\
\pm 6.3 \\
-6.3\end{array}$ & $\begin{array}{l}659 \\
647 \\
725\end{array}$ & & $\begin{array}{l} \pm 6.3 \\
+11.9 \\
-8.5\end{array}$ \\
\hline Post & & & $\begin{array}{r}\text { C } \\
\text { D } \\
P D\end{array}$ & $\begin{array}{l}208 / 108 \\
221 / 120 \\
218 / 113\end{array}$ & $\begin{array}{l}424 \\
399 \\
443\end{array}$ & $\begin{array}{l}90 \\
83 \\
94\end{array}$ & $\begin{array}{l}0.213 \\
0.207 \\
0.212\end{array}$ & $\begin{array}{l} \pm 7.7 \\
+6.0 \\
+4.5\end{array}$ & $\begin{array}{l} \pm 5.7 \\
\pm 8.7 \\
+3.9\end{array}$ & $\begin{array}{l} \pm 5.7 \\
\pm 2.8 \\
+0.5\end{array}$ & $\begin{array}{l}771 \\
725 \\
805\end{array}$ & & $\begin{array}{l} \pm 5.7 \\
+15.1 \\
-1.0\end{array}$ \\
\hline
\end{tabular}

* Proc.-procedure. C-control periods; D-discussion periods; PD-post-discussion periods. B.P.-blood pressure in $\mathrm{mm}$. Hg; EPF-effective plasma flow; GFR-glomerular filtration rate; FF-filtration fraction; EBF-effective blood flow; PRU-peripheral resistance units.

that the hypertensive group does react to a greater degree.

It is of interest that during the periods of relaxation indicated as "post-discussion periods," values still deviated from the control values by amounts of significant size, for effective plasma flow in both groups and for filtration fraction in the hypertensive group in addition. This was in most cases at least 30 minutes after the end of the interview. In general, during the period of emotional stress, and in some cases for considerable time thereafter, there was a change in the renal hemodynamics, characterized by a fall in effective plasma flow, insignificant changes in glomerular filtration rate and a rise in the filtration fraction. When the individual cases are examined and the standard deviations are consulted it is seen that there was more uniformity in this change in filtration fraction than in the others; the variations in renal plasma flow and glomerular filtration rate cooperated more consistently to produce a rise in the filtration fraction. This is shown in Figures 
TABLE II

Comparison of control values and changes in clearances, blood pressures, and vascular resistances in the normotensive and hypertensive groups

\begin{tabular}{|c|c|c|c|}
\hline Average Values & Normotensive & Hypertensive & "P" \\
\hline $\begin{array}{l}\text { Average, Control Periods } \\
\text { Mean blood pressure }(\mathrm{mm} . \mathrm{Hg}) \\
\text { Effective plasma flow }(\mathrm{cc} . / \mathrm{min} .) \\
\text { Glomerular filtration rate }(c c . / \text { min. }) \\
\text { Filtration fraction } \\
\text { Peripheral resistance }(\mathrm{mm} . \mathrm{Hg} / \mathrm{cc} . / \min .)\end{array}$ & $\begin{array}{l}93.5 \pm 9.9 \\
509.0 \pm 94.2 \\
89.1 \pm 11.9 \\
0.176 \pm 0.021 \\
0.098 \pm 0.034\end{array}$ & $\begin{array}{l}137.3 \pm 19.4 \\
460.4 \pm 110.3 \\
102.5 \pm 23.4 \\
0.225 \pm 0.035 \\
0.171 \pm 0.054\end{array}$ & $\begin{array}{l}<0.01 \\
<0.3 \\
<0.05 \\
<0.001 \\
<0.001\end{array}$ \\
\hline 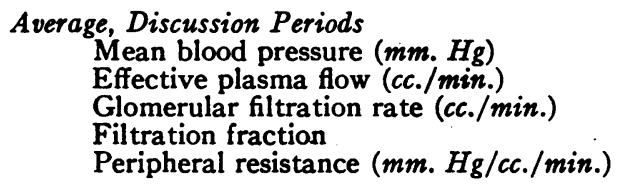 & $\begin{array}{l}102.6 \pm 12.8 \\
482.3 \pm 77.3 \\
88.8 \pm 13.2 \\
0.186 \pm 0.023 \\
0.122 \pm 0.022\end{array}$ & $\begin{array}{l}151.4 \pm 20.7 \\
418.0 \pm 99.1 \\
102.6 \pm 26.8 \\
0.247 \pm 0.030 \\
0.212 \pm 0.066\end{array}$ & $\begin{array}{l}<0.001 \\
<0.05 \\
<0.1 \\
<0.001 \\
<0.001\end{array}$ \\
\hline $\begin{array}{l}\text { Average, Post-discussion Periods } \\
\text { Mean blood pressure }(\operatorname{mm} . \mathrm{Hg}) \\
\text { Effective plasma flow }(\mathrm{cc} . / \mathrm{min} .) \\
\text { Glomerular filtration rate }(c c . / \min .) \\
\text { Filtration fraction } \\
\text { Peripheral resistance }(\mathrm{mm} . \mathrm{Hg} / \mathrm{cc} . / \mathrm{min} .)\end{array}$ & $\begin{aligned} 97.8 & \pm 10.4 \\
493.2 & \pm 84.8 \\
88.3 & \pm 18.1 \\
0.180 & \pm 0.022 \\
0.112 & \pm 0.021\end{aligned}$ & $\begin{array}{c}137.5+16.9 \\
414.2+81.6 \\
99.9+26.2 \\
0.241+0.038 \\
0.186+0.048\end{array}$ & $\begin{array}{l}<0.001 \\
<0.01 \\
<0.2 \\
<0.001 \\
<0.001\end{array}$ \\
\hline Average Changes & Normotensive & Hypertensive & "P" \\
\hline $\begin{array}{l}\text { Discussion-Control Periods } \\
\text { Mean blood pressure }(\mathrm{mm} . \mathrm{Hg}) \\
\text { Effective plasma flow }(c c . / m i n .) \\
\text { Glomerular filtration rate }(c c . / m i n .) \\
\text { Filtration fraction } \\
\text { Peripheral resistance }(\mathrm{mm} . \mathrm{Hg} / \mathrm{cc} . / \mathrm{min} .)\end{array}$ & $\begin{array}{c}+9.3 \pm 5.7 \\
(<0.01) \\
-26.5 \pm 30.1 \\
(<0.01) \\
-0.8 \pm 5.7 \\
(-0.011 \pm 0.009 \\
(0.001) \\
+0.014 \pm 0.012 \\
(<0.001)\end{array}$ & $\begin{array}{c}+17.3 \pm 10.8 \\
(<0.001) \\
-42.4 \pm 52.6 \\
(<0.001) \\
+0.0 \pm 6.9 \\
+0.022 \pm 0.018 \\
(0.001) \\
+0.041 \pm 0.026 \\
(<0.001)\end{array}$ & $\begin{array}{l}<0.02 \\
- \\
- \\
<0.05 \\
<0.01\end{array}$ \\
\hline $\begin{array}{l}\text { Post-discussion-Control Periods } \\
\text { Mean blood pressure }(\mathrm{mm} . \mathrm{Hg}) \\
\text { Effective plasma flow }(c c . / m i n .) \\
\text { Glomerular filtration rate }(c c . / m i n .) \\
\text { Filtration fraction } \\
\text { Peripheral resistance }(\mathrm{mm} . \mathrm{Hg} / c c . / \min .)\end{array}$ & $\begin{array}{c}+2.7 \pm 6.4 \\
(<0.2) \\
-22.7 \pm 30.4 \\
(<0.02) \\
-0.6 \pm 2.3 \\
(\bar{c}) \\
+0.005 \pm 0.013 \\
(<0.2) \\
+0.005 \pm 0.010 \\
(<0.1)\end{array}$ & $\begin{array}{c}+3.0 \pm 7.1 \\
(<0.1) \\
-34.5 \pm 49.6 \\
(<0.01) \\
-0.2 \pm 6.6 \\
(-0.015 \pm 0.021 \\
+(<0.01) \\
+0.017 \pm 0.026 \\
(<0.01)\end{array}$ & $\begin{array}{l}- \\
- \\
- \\
<0.2 \\
<0.2\end{array}$ \\
\hline
\end{tabular}

a) The normotensive group consisted of 13 subjects. The hypertensive group consisted of 20 to 22 subjects. This variation was taken into consideration in the calculation of " $t$ ". Each figure is followed by its standard deviation.

b) " $P$ " represents the degree of probability that the two figures on the same horizontal row are not significant, and was obtained from the tables of distribution after calculation of " $t$ ". The omissions signified by dashes indicate that " $P$ " was greater than 0.2 . Levels of 0.01 or less are to be considered significant.

$$
\begin{aligned}
& t=\left(\bar{X}_{1}-\bar{X}_{2}\right) \cdot \sqrt{\frac{n_{1}-n_{2}\left(n_{1}+n_{2}-2\right)}{\left(n_{1}+n_{2}\right)\left(\Sigma x_{1}+\Sigma x_{2}\right)}} \\
& \Sigma x=\Sigma X^{2}-\frac{(\Sigma X)^{2}}{n}
\end{aligned}
$$

c) The figures in parentheses beneath each value represent " $P$ " with the same significance as under (b), regarding the respective average differences immediately above. " $t$ " was calculated as follows:

$$
t=\frac{\bar{X}}{\text { S.D. } / \sqrt{n}} \quad \text { S.D. }=\sqrt{\frac{X^{2}-\frac{(\Sigma X)^{2}}{n}}{n-1}}
$$




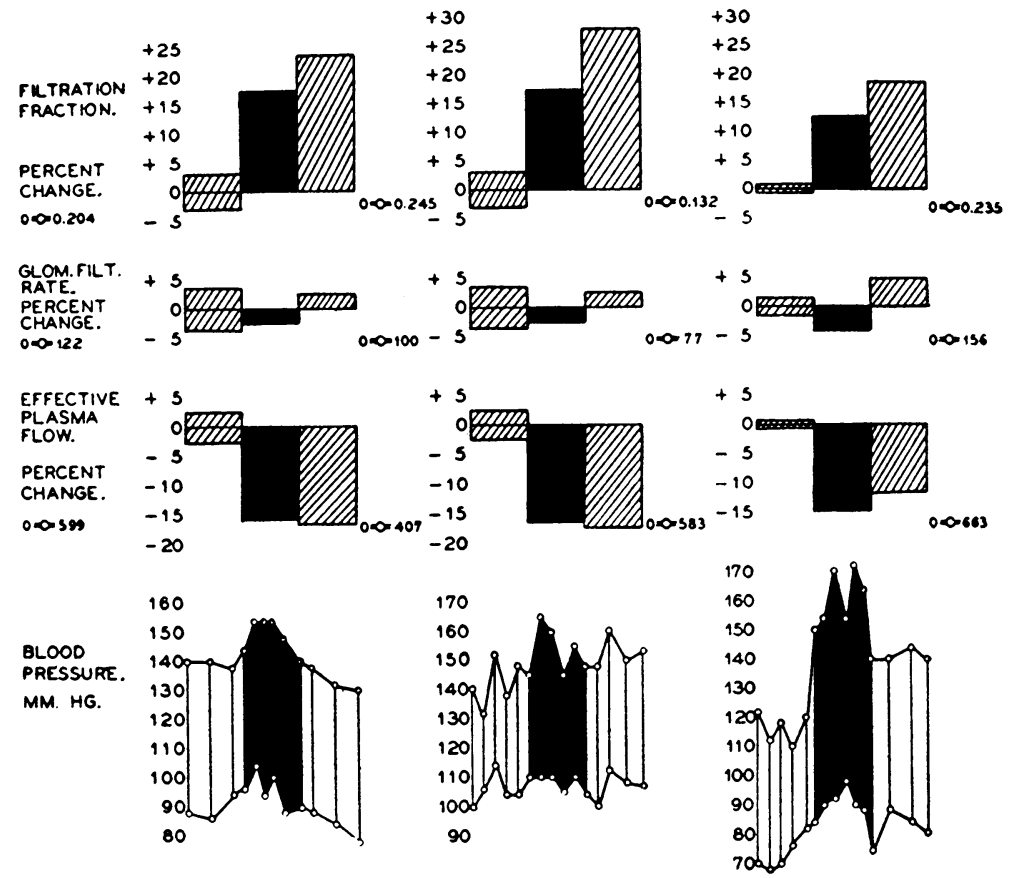

Fig. 1. Effect of Emotional Stress upon Effective Plasma Flow, Glomerular Filtration Rate, Filtration Fraction and Vascular Rrsistance during Elevation of Blood Pressure (Subjects No. 19, No. 6, No. 24). (Black Areas Represent Periods of Discussion)

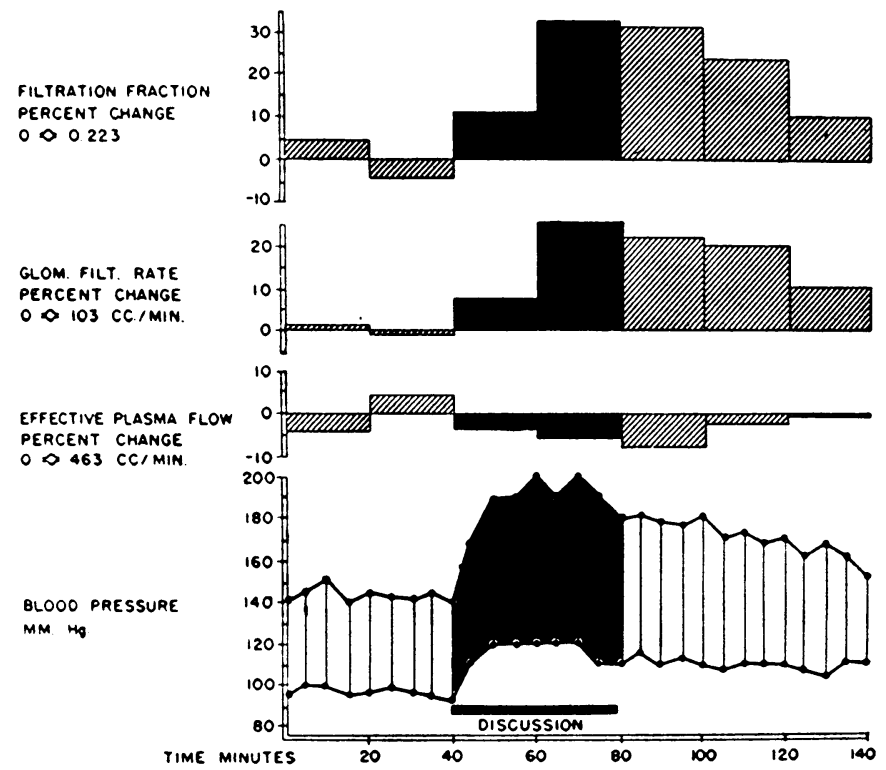

Fig. 2. Prolonged Elevation of Glomerular Filtration Rate with Rise in Filtration Fraction during Elevation of Blood Pressure during Emotional Stress. (Subject 16) 
1 and 2 . In addition, it can be seen that the variations did not necessarily parallel the blood pressure response, the renal hemodynamics being disturbed long after the latter had returned to normal.

Utilizing the ratio of mean blood pressure to effective blood flow (corrected for body surface area) as a crude indication of the vascular resistance of the kidney, other inferences were made possible. In both groups there were large and significant rises in renal vascular resistance; $0.014 \pm 0.012$ units in the normotensive group and $0.041 \pm 0.026$ units in the hypertensive group. As might be expected the control values in the latter group were considerably higher to start with. The tendency to prolongation of the increase in renal vascular resistance is indicated by the length of time for the figures to return to control values (Table II and Figure 3 ). Thus in both normotensives and hypertensives the kidney adjusted itself by means of vasoconstriction to the rise in systemic blood pressure, preventing increase of blood flow through it. There is suggestive evidence that the hypertensive kidney overcompensates so that it exhibits both a more intense and a more prolonged constriction, even

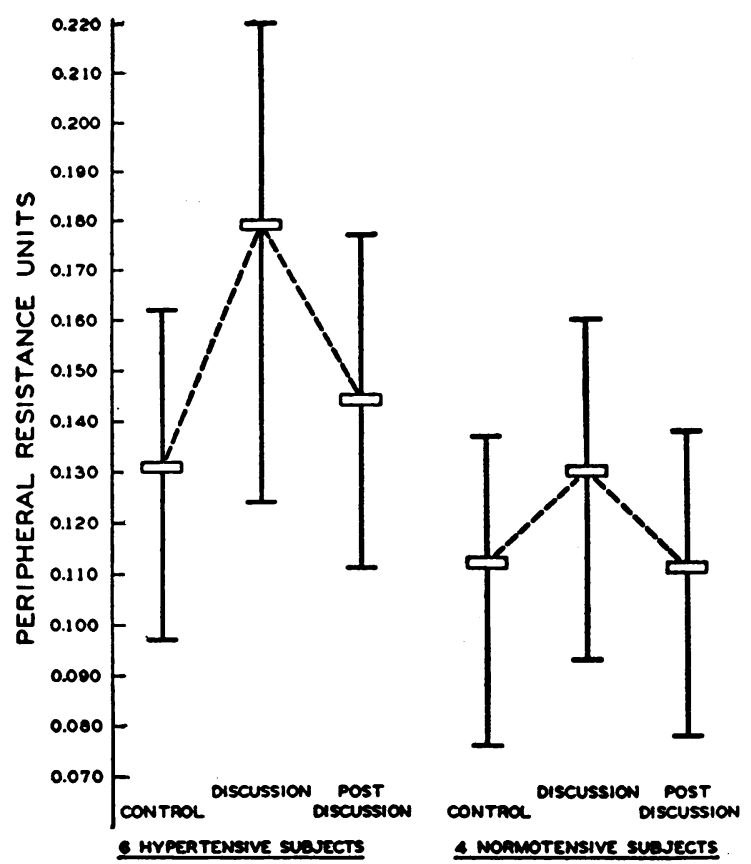

Fig. 3. Averaged Changes in Renal Vascular Resistance during Control Periods, during Periods of Stressful Interviews and during the Period IMmediately after Stress in Six Hypertensive Subjects as Compared to Four Normotensive Subjects

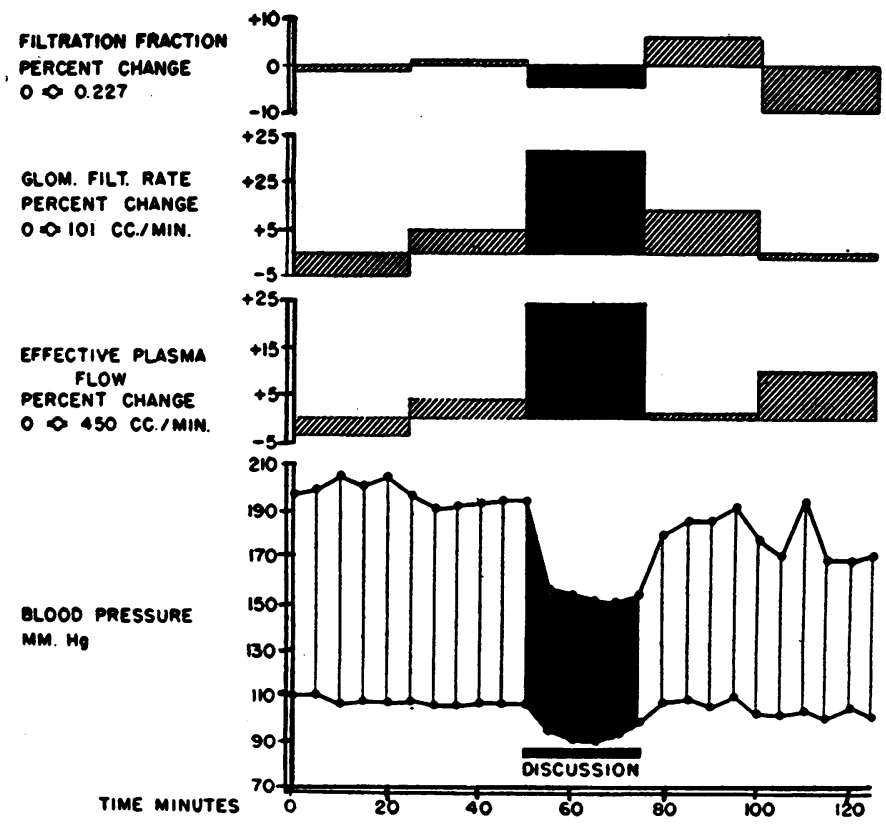

Fig. 4. Effect of Relaxation Associated with Fall in Brood Pressure on Emfective Plasma Flow, Glomerular Filtration Rate and Futration Fraction 
after the blood pressure has returned to near control levels.

\section{B. Variations in blood pressure, effective plasma flow, glomerular filtration rate and renal vascular resistance during relaxation}

The converse of the above experiments was inadvertently exhibited, when, in attempting to restrict the sphere of attention by the use of sodium amytal (19), one hypertensive subject became very relaxed and indulged in pleasurable phantasies before the interview was started. An effort was made to promote and maintain this relaxation. The blood pressure fell from an average control level of $197 / 109$ to $152 / 92$ during the period of relaxation. During the fall in blood pressure there was a rise in effective plasma flow of $24 \%$ and in glomerular filtration rate of $19 \%$ with a resultant fall in filtration fraction (Table I, No. 36 and Figure 4). At the end of the clearance period the subject was aroused from his pleasurable relaxation and the blood pressure returned sharply to control levels, as did the other values.

Comment. The observation that arterial pressure and renal clearances may change toward normal during relaxation, supports the view that the rise in pressure and fall in clearances during the stressful interview situation are truly reactions to stress and suggests in addition that the subject with essential hypertension may well be living in a sustained state of overreaction to the minor stresses of daily life.

\section{Effect of lumbodorsal sympathectomy and splanchnicectomy in renal vascular func- tion during emotional stress}

Following lumbodorsal sympathectomy and splanchnicectomy performed upon six of the subjects with essential hypertension, the entire experimental procedure as described above was repeated. A considerable rise in blood pressure was obtained in each of the subjects post-operatively during the discussion of disturbing topics. In two of these subjects (Figure 5, Subject 20), the changes in the renal vascular function were the complete reverse of those observed before the operation, i.e., with the rise in blood pressure, there was a rise instead of a fall in effective plasma flow and a fall instead of a rise in the filtration fraction (Table I). In the others, there was a partial inversion of the pattern, consisting either in a failure of the filtration fraction to rise or of the effective plasma flow to fall. Thus, in all six of these subjects, there was evidence that the renal vascular resistance was altered during the period of rise in blood pressure. This was considered as evidence that, in the absence of the renal nerve supply so far as could be determined, the kidney nevertheless continued to control its blood flow. In an attempt to determine more fully where the site of this resistance was located, the data available from all the subjects, with and without sympathectomy were re-evaluated by means of the formulae of Lamport $(16,20-22)$ for the calculation of efferent and afferent arteriolar resistance. The results of these calculations (Table III) indicated that there was a rise in both afferent and efferent arteriolar resistance in all 35 subjects without sympathectomy. Following the operative procedure in five of the six subjects, however, although the afferent arterioles continued to constrict during the blood pressure rise, the efferent arteriolar re-

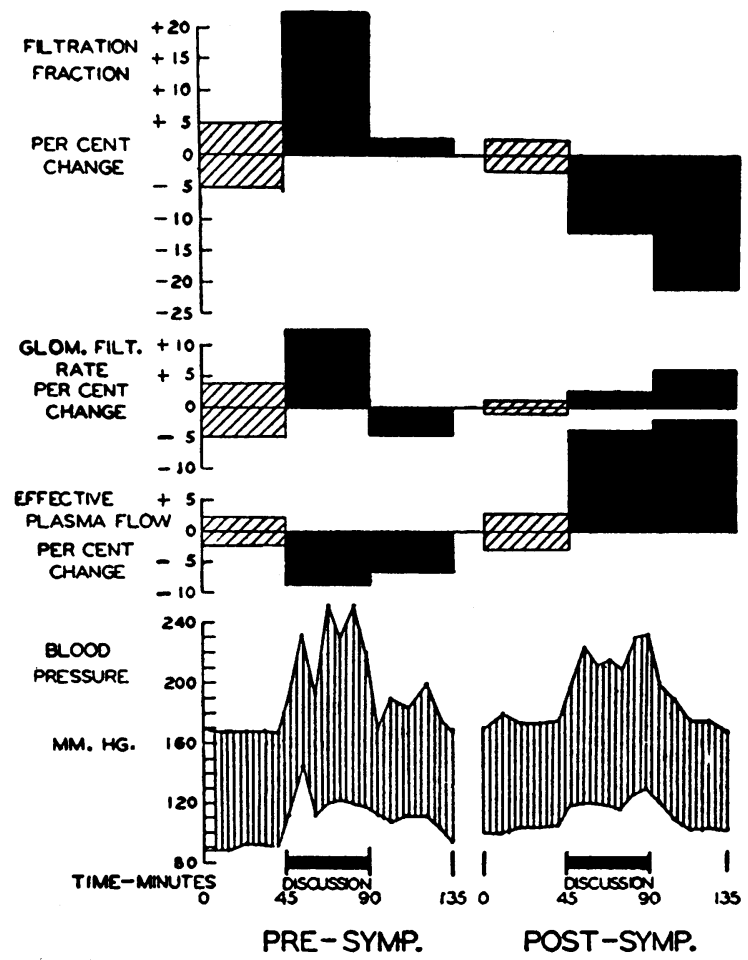

Fig. 5. Changes in Pattern of Reaction of Renal. Clearances before and after Sympathectomy. (SubJECT 20) 
sistance was definitely inhibited. It is of interest that the subject in whom this phenomenon was not successfully demonstrated, had a partial sympathectomy; the lower portion of the sympathetic chain, L-2, was not identified in the specimen examined post-operatively and presumably had not been removed as intended. For comparison, be- cause Gomez $(17,18)$ has developed a different mathematical treatment of renal hemodynamics, efferent arteriolar resistence was calculated by his method. The results are also shown in Table III and are comparable to those obtained with Lamport's formulae, both in magnitude and direction of change.

TABLE III

Summary of afferent and efferent arteriolar resistance pre- and post-sympathectomy*

\begin{tabular}{|c|c|c|c|c|c|c|c|c|c|c|}
\hline \multirow{2}{*}{\multicolumn{2}{|c|}{ Pt. }} & \multirow{2}{*}{$\begin{array}{l}\text { Aver. } \\
\text { MBP }\end{array}$} & \multicolumn{6}{|c|}{ Calculations by Lamport's Formulae } & \multicolumn{2}{|c|}{ by Gomez' Formulae } \\
\hline & & & $1000 \times R \mathbf{R}$ & $1000 \times$ Re & $1000 \times R t$. & $\Delta \% \mathbf{R a}$ & $\Delta \% \operatorname{Re}$ & $\Delta \%$ Rt. & $\operatorname{Re} \times \mathbf{K}$ & $\Delta \% \operatorname{Re} \times \mathrm{K}$ \\
\hline $\begin{array}{c}17 \text { R. W. } \\
\text { Pre }\end{array}$ & $\begin{array}{r}\mathrm{C} \\
\mathrm{D} \\
\mathrm{PD}\end{array}$ & $\begin{array}{l}119 \\
142 \\
115\end{array}$ & $\begin{array}{l}42.0 \\
77.9 \\
46.1\end{array}$ & $\begin{array}{l}17.9 \\
25.8 \\
29.1\end{array}$ & $\begin{array}{r}59.9 \\
103.7 \\
75.2\end{array}$ & $\begin{array}{r}\overline{85.5} \\
+\quad 9.8\end{array}$ & $\begin{array}{r}-\overline{44} .1 \\
+62.6\end{array}$ & $\begin{array}{r}\overline{73} .0 \\
+25.5\end{array}$ & $\begin{array}{l}0.116 \\
0.148 \\
0.160\end{array}$ & $\begin{array}{r}\overline{27.6} \\
+37.9\end{array}$ \\
\hline Post & $\begin{array}{r}C \\
D \\
P D\end{array}$ & $\begin{array}{l}123 \\
141 \\
124\end{array}$ & $\begin{array}{l}53.9 \\
79.3 \\
55.7\end{array}$ & $\begin{array}{l}25.7 \\
25.8 \\
22.1\end{array}$ & $\begin{array}{r}79.6 \\
105.1 \\
77.8\end{array}$ & $\begin{array}{r}-\overline{47.2} \\
+\quad 3.3\end{array}$ & $\begin{array}{r}-\overline{0.4} \\
-14.0\end{array}$ & $\begin{array}{r}\overline{35.0} \\
+\quad 2.3\end{array}$ & $\begin{array}{l}0.157 \\
0.155 \\
0.124\end{array}$ & $\begin{array}{r}-1.3 \\
-22.0\end{array}$ \\
\hline 20 M. G. & $\begin{array}{r}\mathrm{C} \\
\mathrm{D} \\
\mathrm{PD}\end{array}$ & $\begin{array}{l}122 \\
160 \\
145\end{array}$ & $\begin{array}{r}57.7 \\
109.0 \\
87.1\end{array}$ & $\begin{array}{l}18.2 \\
22.6 \\
19.7\end{array}$ & $\begin{array}{r}75.9 \\
131.6 \\
106.8\end{array}$ & $\begin{array}{r}-\overline{88.8} \\
+33.6\end{array}$ & $\begin{array}{r}\overline{24.2} \\
+8.2\end{array}$ & $\begin{array}{r}-\overline{73.4} \\
+40.7\end{array}$ & $\begin{array}{l}0.110 \\
0.140 \\
0.115\end{array}$ & $\begin{array}{r}\overline{27.3} \\
+4.5\end{array}$ \\
\hline Post & $\begin{array}{r}\mathrm{C} \\
\mathbf{P D}\end{array}$ & $\begin{array}{l}135 \\
158 \\
134\end{array}$ & $\begin{array}{l}112.4 \\
147.0 \\
103.0\end{array}$ & $\begin{array}{l}49.1 \\
38.6 \\
34.2\end{array}$ & $\begin{array}{l}116.5 \\
184.6 \\
137.2\end{array}$ & $\begin{array}{r}\overline{30.8} \\
-8.4\end{array}$ & $\begin{array}{r}-\overline{21.4} \\
-30.4\end{array}$ & $\begin{array}{r} \\
+14.3 \\
-15.0\end{array}$ & $\begin{array}{l}0.185 \\
0.160 \\
0.142\end{array}$ & $\begin{array}{r}-\overline{13.5} \\
-23.2\end{array}$ \\
\hline $21 \underset{\text { Pre }}{\mathrm{H} .}$ W. & $\begin{array}{r}\mathrm{C} \\
\mathrm{D} \\
\mathbf{P D}\end{array}$ & $\begin{array}{l}129 \\
146 \\
131\end{array}$ & $\begin{array}{r}85.3 \\
103.1 \\
84.4\end{array}$ & $\begin{array}{l}27.9 \\
30.9 \\
25.9\end{array}$ & $\begin{array}{l}113.2 \\
134.0 \\
110.3\end{array}$ & $\begin{array}{r}\overline{1} .9 \\
+20.9 \\
-1.1\end{array}$ & $\begin{array}{r}-\overline{10.8} \\
-7.2\end{array}$ & $\begin{array}{r}\overline{18.4} \\
-\quad 2.6\end{array}$ & $\begin{array}{l}0.127 \\
0.133 \\
0.121\end{array}$ & $\begin{array}{r}- \\
+4.7 \\
-4.7\end{array}$ \\
\hline Post & $\begin{array}{r}\mathrm{C} \\
\mathrm{PD}\end{array}$ & $\begin{array}{l}139 \\
151 \\
141\end{array}$ & $\begin{array}{l}103.1 \\
122.0 \\
100.3\end{array}$ & $\begin{array}{l}27.1 \\
27.7 \\
25.2\end{array}$ & $\begin{array}{l}130.2 \\
149.7 \\
125.5\end{array}$ & $\begin{array}{r}-\overline{18.3} \\
-2.7\end{array}$ & $\begin{array}{r}-\overline{2.2} \\
+7.0\end{array}$ & $\begin{array}{r}\overline{15.0} \\
-\quad 3.6\end{array}$ & $\begin{array}{l}0.131 \\
0.143 \\
0.127\end{array}$ & $\begin{array}{r}-\overline{9.2} \\
+3.1\end{array}$ \\
\hline $27 \underset{\text { Pre }}{\text { B. } R .}$ & $\begin{array}{r}\mathrm{C} \\
\mathrm{PD}\end{array}$ & $\begin{array}{l}140 \\
163 \\
145\end{array}$ & $\begin{array}{r}77.6 \\
123.0 \\
96.5\end{array}$ & $\begin{array}{l}19.4 \\
31.8 \\
25.0\end{array}$ & $\begin{array}{r}97.0 \\
154.8 \\
121.5\end{array}$ & $\begin{array}{r}-\overline{58.5} \\
+24.4\end{array}$ & $\begin{array}{r}-\overline{63} .9 \\
+28.9\end{array}$ & $\begin{array}{r}\overline{+} \\
+59.5 \\
+25.3\end{array}$ & $\begin{array}{l}0.134 \\
0.155 \\
0.153\end{array}$ & $\begin{array}{r}-\overline{18.7} \\
+14.2\end{array}$ \\
\hline Post & $\begin{array}{r}\mathrm{C} \\
\mathrm{D} \\
\mathrm{PD}\end{array}$ & $\begin{array}{l}103 \\
116 \\
108\end{array}$ & $\begin{array}{l}31.6 \\
48.2 \\
41.1\end{array}$ & $\begin{array}{l}16.9 \\
18.8 \\
19.3\end{array}$ & $\begin{array}{l}48.5 \\
67.0 \\
60.4\end{array}$ & $\begin{array}{r}-\overline{52} .5 \\
+30.1\end{array}$ & $\begin{array}{r}\overline{-} \\
+11.2 \\
+14.2\end{array}$ & $\begin{array}{r}\overline{38.2} \\
+24.6\end{array}$ & $\begin{array}{l}0.119 \\
0.123 \\
0.117\end{array}$ & $\begin{array}{r}-\overline{3.4} \\
+1.7\end{array}$ \\
\hline $30 \underset{\text { Pre }}{\mathrm{E} .} \mathrm{W}$. & $\begin{array}{r}C \\
D \\
P D\end{array}$ & $\begin{array}{l}143 \\
184 \\
154\end{array}$ & $\begin{array}{l}125.6 \\
218.0 \\
153.9\end{array}$ & $\begin{array}{l}35.2 \\
44.5 \\
42.0\end{array}$ & $\begin{array}{l}160.8 \\
262.5 \\
195.9\end{array}$ & $\begin{array}{r}-\overline{74.4} \\
+22.6\end{array}$ & $\begin{array}{r}\overline{26.4} \\
+19.3\end{array}$ & $\begin{array}{r}\overline{+63.2} \\
+21.8\end{array}$ & $\begin{array}{l}0.133 \\
0.163 \\
0.148\end{array}$ & $\begin{array}{r}\overline{22.3} \\
+11.6\end{array}$ \\
\hline Post & $\begin{array}{r}\mathrm{C} \\
\mathrm{D} \\
\mathrm{PD}\end{array}$ & $\begin{array}{l}159 \\
180 \\
160\end{array}$ & $\begin{array}{l}166.1 \\
187.3 \\
153.4\end{array}$ & $\begin{array}{l}36.1 \\
33.8 \\
34.7\end{array}$ & $\begin{array}{l}202.2 \\
221.1 \\
188.1\end{array}$ & $\begin{array}{r}-\overline{12.8} \\
-7.7\end{array}$ & $\begin{array}{r}-\overline{6.4} \\
-3.9\end{array}$ & $\begin{array}{r}- \\
+\quad 9.4 \\
-7.0\end{array}$ & $\begin{array}{l}0.136 \\
0.144 \\
0.139\end{array}$ & $\begin{array}{r}- \\
+5.9 \\
+2.2\end{array}$ \\
\hline $32 \underset{\text { Pre }}{\text { R. L. }}$ & $\begin{array}{r}\mathrm{C} \\
\mathrm{D} \\
\mathbf{P}\end{array}$ & $\begin{array}{r}\cdot 160 \\
174 \\
158\end{array}$ & $\begin{array}{l}148.8 \\
175.3 \\
136.3\end{array}$ & $\begin{array}{l}40.8 \\
44.3 \\
35.1\end{array}$ & $\begin{array}{l}189.6 \\
219.6 \\
171.4\end{array}$ & $\begin{array}{r}\overline{17.8} \\
-8.4\end{array}$ & $\begin{array}{r}-\overline{8.6} \\
-14.0\end{array}$ & $\begin{array}{r}-\overline{15.8} \\
-\quad 9.6\end{array}$ & $\begin{array}{l}0.142 \\
0.152 \\
0.134\end{array}$ & $\begin{array}{r}-\overline{7.0} \\
-7.0\end{array}$ \\
\hline Post & $\begin{array}{r}C \\
D \\
P D\end{array}$ & $\begin{array}{l}152 \\
164 \\
159\end{array}$ & $\begin{array}{l}107.2 \\
133.2 \\
113.0\end{array}$ & $\begin{array}{l}26.4 \\
27.4 \\
25.1\end{array}$ & $\begin{array}{l}133.6 \\
160.6 \\
138.1\end{array}$ & $\begin{array}{r}-\overline{23.3} \\
+\quad 5.4\end{array}$ & $\begin{array}{r}- \\
+3.8 \\
+4.9\end{array}$ & $\begin{array}{r}\overline{2} \\
+20.2 \\
+3.4\end{array}$ & $\begin{array}{l}0.115 \\
0.112 \\
0.115\end{array}$ & $\begin{array}{r}- \\
-2.6 \\
\pm 0.0\end{array}$ \\
\hline
\end{tabular}

- Definition of Symbols

$$
\begin{aligned}
& \mathrm{MBP} \text {-mean blood pressure } \\
& \mathrm{Ra} \text {-afferent arteriolar resistance } \\
& \mathrm{Re} \text {-efferent arteriolar resistance } \\
& \mathrm{Rt} \text {-total renal resistance (equals } \mathrm{Ra} \text { plus } \mathrm{Re} \text { ) } \\
& \mathrm{C} \text {-control period }
\end{aligned}
$$

D -discussion period

PD - post-discussion period

Pre - before sympathectomy

Post-after sympathectomy

$\Delta \%$ - change in per cent 

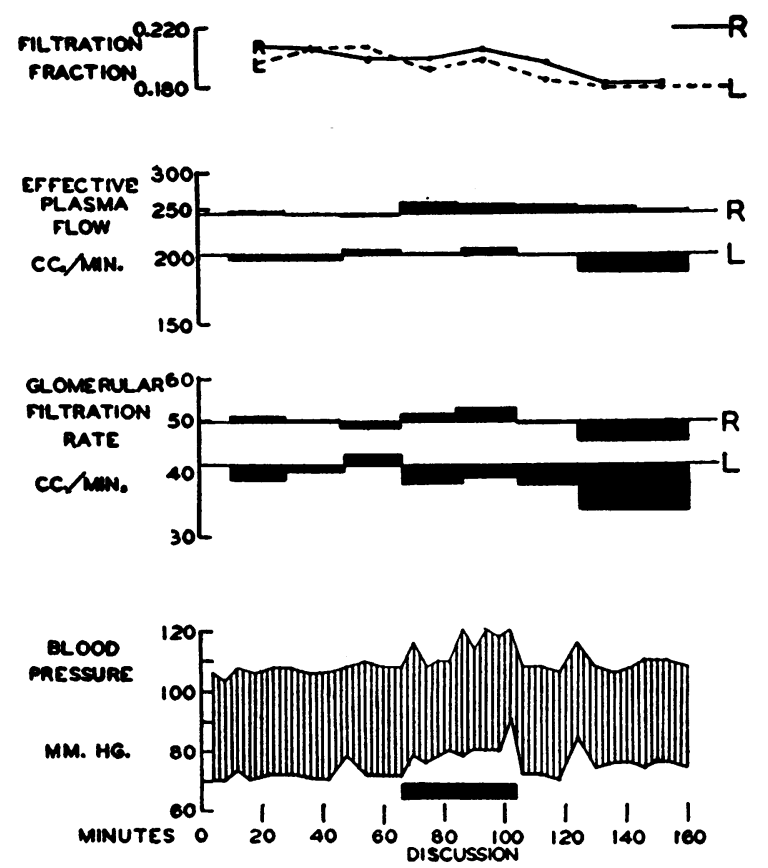

Fig. 6. Clearances on Separate Kidneys in a Subject with Normal Blood Pressure and with Right Lumbodorsal Sympathectomy. (Subject 37)

Comment. It is suggested by these experiments that during the emergency response to stress both afferent and efferent arterioles constrict but that the action of the latter is diminished or abolished after removal of the renal nerve supply by sympathectomy. However, even though the efferent arteriole ceases to function, the afferent arteriole has a certain autonomy of control which enables the kidney to "protect" itself against a systemic rise in blood pressure. The effect of sympathectomy on renal vascular function has been extensively studied by others (2, pp. 155-157) and found to be nil. It is to be emphasized that these latter studies were all done in the basal state, and that our subjects, too, showed no changes unless subjected to stress. This is in keeping with the general experience with this component of autonomic integrative action. As an illustration of this, in Figure 6 are shown the results of study of separate kidneys on a normotensive subject with right unilateral sympathectomy because of pain. The patient was exposed to the discussion of a topic which did not disturb her; there was no response in terms of blood pressure and no significant changes in clearances.

\section{DISCUSSION}

\section{A. Pattern of reaction during stressful situations \\ 1. Blood pressure}

During the course of the experiments, the problem arose as to what criteria must be established in order to distinguish between the so-called hypertensives and the normotensives. The level of blood pressure at any one time was not satisfactory, as evaluation of the clinical records of the subjects

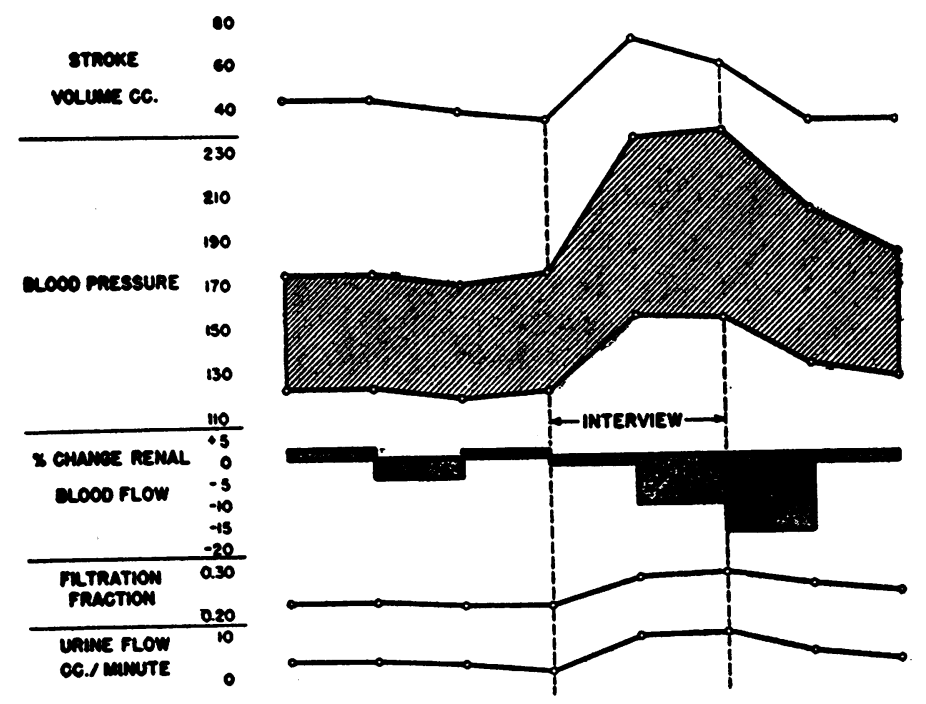

Fig. 7. Changes in Cardiac Output and Renal Dynamics during Emotional Stress. (Subject 30) 
studied showed that occasional elevated blood pressures were exhibited by the group characterized as having "normal" blood pressures and in like manner, occasional pressures within the accepted normal range were exhibited by the essential hypertension group. It did not appear that there was validity in attempting to distinguish clearly between the "normal individual" with a hyperactive vascular system and the "abnormal individual" with essential hypertension inasmuch as this very point is the subject of considerable discussion. It is possible that the differences are merely quantitative in the early phases of the hypertensive disease and only become qualitative as various organs and organ systems succumb to the stress imposed. Regarding the pressor responses in the subjects studied herein, statistical analysis indicates that there is only one chance in 50 that the degree of pressor response to emotional stress in the hypertensive group might be duplicated in the normotensive group in similar additional trials.

Studies on cardiac output in subjects with essential hypertension have shown that the resting cardiac output is within normal limits (23) and that the ratio of renal blood flow to cardiac index (liters cardiac output/1.73 sq. m. body area) was low (24). In two of the subjects reported in this study (Figure 7) simultaneous measurements of cardiac output (by means of the ballistocardiograph) and of renal blood flow were made, and in 35 additional patients, 19 hypertensives and 16 normotensives, measurements of cardiac output alone were made by Dr. Ian Stevenson (23) during induced emotional conflict and anxiety. During such induced stress, the blood pressure rise in the two groups was comparable to that noted in our subjects, namely, 17 and $8 \mathrm{~mm}$. $\mathrm{Hg}$ in hypertensives and normotensives, respectively. In these circumstances of increase in blood pressure, the cardiac output was found to increase $42 \%$ and $53 \%$, respectively, but the peripheral resistance decreased $20 \%$ in the hypertensives and $32 \%$ in the normotensives. There was a $16 \mathrm{~mm}$. $\mathrm{Hg}$ increase in blood pressure per liter increase in the cardiac index in the hypertensive group as compared to $4 \mathrm{~mm}$. $\mathrm{Hg}$ increase in blood pressure per liter increase in cardiac index in the normotensive group.

The blood pressure in the hypertensives during these interview situations remains elevated for a longer time than does the cardiac output which falls rather rapidly to control levels following the cessation of the interview. Consequently, the overall peripheral resistance rises secondarily and it alone accounts for the elevation in blood pressure at this time. It is possible that increase in cardiac output in comparison to the rise in peripheral resistance may vary in amount in different hypertensive subjects. In those with a sustained increase in cardiac output the heart would presumably suffer, whereas the organs implicated in increasing peripheral resistance, such as the kidney, would be damaged by the vascular effort to maintain the elevated blood pressure.

\section{Renal hemodynamics}

The changes in the hemodynamics of the renal circulation during certain varieties of emotional conflict, as indicated by decrease in renal blood flow, unchanged glomerular filtration rate and rise in filtration fraction, are very similar to those known to occur following the administration of epinephrin (5) and are also an intensification of the pattern characteristic of essential hypertension (25). It is clear that these changes are of considerable statistical significance but it is less clear that there is a quantitative difference between the hypertensive and normotensive groups, except in the degree of increase in renal vascular resistance. From the analyses of Smith and associates (26), it would appear that this effect is due predominantly to constriction of the efferent glomerular arteriole. Lamport (16, 20-22), after deriving formulae for afferent and efferent arteriolar resistances in a somewhat different fashion, stated that the afferent arterioles were also involved in this vasoconstriction. Calculations based on our data, using his formulae, indicated a considerable increase in afferent as well as efferent arteriolar resistance during the rise in blood pressure in individuals with intact sympathetic nervous systems.

\section{B. Mechanism of control of renal blood flow}

The mechanism producing the increased blood pressure in responses of this type and in essential hypertension has been the subject of intensive investigation. Evidence has been educed both to 
implicate the kidney in the genesis of the elevated pressure ( 7 ) and, on the other hand, to indicate that the kidney is a passive sufferer from systemic vascular disease (2). Our studies shed no light upon this problem except insofar as they indicate a more intense renal vascular activity in the hypertensive which might in itself be damaging to the kidney.

On the basis of our studies, it appears likely that the sympathetic nervous system controls the efferent glomerular arteriole. There is evidence from animal experiments performed by others that the sympathetic nervous system does have such an action. Burton-Opitz and Lucas (27) showed that stimulation of the splanchnic nerves caused a fall in the renal blood flow measured by means of the thermostromuhr. Incidentally, these investigators were unable to demonstrate an effect of the vagus on renal blood flow (28). Weak splanchnic stimulation has been shown by Winton (29) and small doses of epinephrin by Richards and Plant (30) and Livingston (31) to cause both an increase in the volume of the kidney and a decrease in renal blood flow. These facts add weight to the concept that the adrenergic system of nerves, under circumstances comparable to the more usual physiological responses, are involved in the function of the efferent glomerular arteriole. Stronger stimulation (30) and larger doses of epinephrin (31) produce a decrease in the size of the kidney and a further fall in renal blood flow, indicating that the afferent glomerular arteriole also responds to circulating humoral agents of epinephrin type, but only in larger amounts. More recently Kottke and his colleagues $(32,33)$ by stimulation of the renal nerves for both short and prolonged periods in animals, produced a decrease in renal blood flow and a renal hemodynamic pattern identical with that in essential hypertension and in these studies. A severe hypertension was produced in addition.

If it be accepted that the efferent glomerular arteriole is under control of the sympathetic nervous system, based on the evidence of the failure of efferent arteriolar constriction after sympathectomy, there remains to be explained how the renal blood flow was maintained at a relatively constant level in our experiments after presumed renal denervation. There is a large body of evidence very adequately summarized by Smith (6, pp. 169-175), that there is located in the kidney itself an apparatus which may be involved in the control of renal blood flow. The juxta-glomerular apparatus described consists of a heaping up of the distal convoluted tubular epithelium adjacent to the afferent arteriole, at a point just before the entrance of the latter into the glomerulus, and certain modifications of the muscle fibers of the arteriole itself at the same point. In experimental renal hypertension and in ischemia associated with the "crush syndrome," changes have been observed in this apparatus (34). It is postulated that it is this apparatus which takes over the control of renal blood flow in the denervated kidney and in our sympathectomized patients to "protect" the kidney against the rises in systemic pressure.

In the control of the renal circulation, it is suggested that the two systems of arterioles in the kidney are under control of two different mechanisms; the efferent arteriole is under the control of the sympathetic nervous system and is responsive to impulses arriving by this route, whatever might be the stimulus setting off the discharge within the autonomic nervous system; the afferent arteriole is under the control of an intrinsic autonomous mechanism, presumably humoral, which acts to "protect" the kidney, especially the glomerulus, against rises in systemic pressure, to readjust intraglomerular pressure associated with changes in efferent arteriolar resistance, and perhaps to respond to certain unknown circulating humoral factors. More recently Trueta and his associates (35), in his studies of intrarenal shunt mechanisms, indicates that afferent arteriolar vasoconstriction is an important factor in the shunting of the blood from the cortex to the corticomedullary junction.

\section{Implications of this pattern of response during emotional stress in the hypertensive}

The hypertensive with his heightened reactivity of renal vasculature constricts his renal vessels more intensely and longer than the normotensive in similar situations of stress. Furthermore, if such an intrarenal shunt mechanism as Trueta described be operative in the human kidney, the renal cortex may be more severely ischemic than is indicated by the depression of renal blood flow as measured by the clearance methods. The possibility of renal ischemia becomes especially pertinent inasmuch as available studies indicate that 
the subject with essential hypertension exhibits evidence of sustained strain of emotional character $(1,9-11,19,23)$.

\section{SUMMARY AND CONCLUSIONS}

1. Renal blood flow and filtration rate were studied in 35 subjects with and without arterial hypertension during periods of elevation in both systolic and diastolic blood pressure induced by discussion of important personal topics having a threatening significance.

2. During such rises in blood pressure, there was a constant fall in effective renal blood flow and rise in filtration fraction, indicating an increase in the resistance offered by the renal arterioles.

3. The changes in renal blood flow, filtration fraction and vascular resistance were similar in direction in both the hypertensive and normotensive groups. There was an indication that the decrease in renal blood flow and filtration fraction was more severe in the hypertensive group. The increase in renal vascular resistance was unequivocally greater in the hypertensive group.

4. Following lumbodorsal sympathectomy and splanchnicectomy, induced elevations in systemic arterial pressure were accompanied by a reversal of the above pattern, i.e., a fall in filtration fraction and a less intense renal vascular constriction. This is interpreted as a failure, in whole or part, of the efferent glomerular arteriole to participate, although the afferent arteriole is still responsive.

5. The concept is advanced that the kidney, under usual conditions has two more or less independent vascular mechanisms within it, (a) a sympathetic control of the efferent glomerular arteriole and $(b)$ an intrinsic or intrarenal humoral control of the afferent glomerular arteriole, possibly involving the juxta-glomerular myoarterial apparatus.

\section{BIBLIOGRAPHY}

1. Wolf, S., Pfeiffer, J. B., Ripley, H. S., Winter, O. S., and Wolf, $H$. G., Hypertension as a reaction pattern to stress; summary of experimental data on variations in blood pressure and renal blood flow. Ann. Int. Med., 1948, 29, 1056.

2. Goldring, W., and Chasis, H., Hypertension and $\mathrm{Hy}-$ pertensive Disease. The Commonwealth Fund, New York, 1944.

3. Wolf, G. A., Effect of pain on renal function. Research in Nerv. \& Ment. Dis., Proc., 1943, 23, 358.
4. Talso, P. J., Crosley, A. P., Jr., and Clarke, R. W., Effects of the cold pressor test on glomerular filtration and effective renal plasma flow. Federation Proc., 1948, 7, 122.

5. Ranges, H. A., and Bradley, S. E., Systemic and renal circulatory changes following the administration of adrenin, ephedrine and paredrinal to normal men. J. Clin. Invest., 1943, 22, 687.

6. Smith, H. W., Physiology of the renal circulation. Harvey Lecture, 1939-40, pp. 204-207.

7. Goldblatt, H., The renal origin of hypertension. Physiol. Rev., 1947, 27, 120.

8. O'Hare, J. P., Vascular reactions in essential hypertension. Am. J. M. Sc., 1920, 159, 369.

9. Fahrenkamp, K., Die Psycho-physichen Wechselwirkungen bei den Hypertoniekrankungen. Eine klinische Studie über die praktische Bedeutung der Blutdruckkurve. Hippokrates Verlag, Stuttgart, 1926.

10. Saul, L. J., Hostility in cases of essential hypertension. Psychosomatic Med., 1939, 1, 153.

11. Alexander, F., Emotional factors in essential hypertension, presentation of tentative hypothesis. Psychosomatic Med., 1939, 1, 173.

12. Smithwick, R. H., Surgical treatment of hypertension. The effect of radical splanchnicectomy on the hypertensive state of 156 patients followed for one to five years. Arch. Surg., 1944, 49, 180.

13. Hare, K. E., Personal Communication.

14. Hubbard, R. S., and Loomis, T. A., Determination of inulin. J. Biol. Chem., 1942, 145, 641.

15. Corcoran, A. C., and Page, I. H., Method for the determination of mannitol in blood and urine. Federation Proc., 1946, 5, 130.

16. Lamport, H., Formulae for afferent and efferent arteriolar resistance in the human kidney. An application to the effects of spinal anesthesia. J. Clin. Invest., 1941, 20, 535.

17. Gomez, D. M., Hemodynamics of the renal circulation. La Rev. Scientifique, 1947, 3272, 451.

18. Gomez, D. M., Calculation of effective afferent and efferent arteriolar resistance. Federation Proc., 1948, 7, 41.

19. Ripley, H. S., and Wolf, S., The intravenous use of sodium amytal in psychosomatic disorders. Psychosomatic Med., 1947, 9, 260.

20. Lamport, $H$., The relative change in afferent and efferent arteriolar resistance in the normal human kidney. J. Clin. Invest., 1941, 20, 545.

21. Lamport, H., Effect on renal resistance to blood flow of renin, angiotonin, pitressin, atropin, hypertension and toxemia of pregnancy. J. Clin. Invest., 1942, 21, 685.

22. Lamport, H., Improvements in calculation of renal resistance to blood flow. Charts for osmotic pressure and viscosity of blood. J. Clin. Invest., 1943, 22, 461.

23. Stevenson, I. P., Duncan, C. H., and Wolf, H. G., Circulatory dynamics before and after exercise in 
subjects with and without structural heart disease during anxiety and relaxation. J. Clin. Invest., 1949, 28, 1534.

24. Bolomey, A. A., Breed, E. F., Michie, A., Michie, K., and Lawson, $H$. D., Renal function in normal subjects and subjects with essential hypertension. Federation Proc., 1948, 7, 10.

25. Goldring, W., Chasis, H., Ranges, H. A., and Smith, H. W., Effective renal blood flow in subjects with essential hypertension. J. Clin. Invest., 1941, 20, 637.

26. Smith, H. W., Chasis, H., Goldring, W., and Ranges, H. A., Glomerular dynamics in the normal human kidney. J. Clin. Invest., 1940, 19, 751.

27. Burton-Opitz, R., and Lucas, D. R., Utber die Blutversorgung der Niere. Der Einfluss der Erhöhung des Druckes in den Narnwegen sowie der Reizung und Durchschneidung der den Plexus renalis bildenden Nervenfasern. Arch. f. d. ges Physiol., 1908, 123, 553.

28. Burton-Opitz, R., and Lucas, D. R., The blood supply of the kidney. V. The influences of the vagus nerve upon the vascularity of the left organ. J. Exper. Med., 1911, 13, 308.
29. Winton, F. R., Physical factors involved in the activities of the mammalian kidney. Physiol. Rev., 1937, 17, 408.

30. Richards, A. N., and Plant, O. H., The action of minute doses of adrenalin and pituitrin on the kidney. Am. J. Physiol., 1922, 59, 191.

31. Livingston, A. E., Further experiments on the effects of small doses of vasoconstrictor substances on the kidney. J. Pharmacol. \& Exper. Therap., 1928, 32, 181.

32. Kottke, F. J., Kubicek, W. G., and Laker, D. J., Physical and nervous factors in experimental hypertension. Arch. Phys. Med., 1947, 28, 146.

33. Kottke, F. J., Kubicek, W. G., and Visscher, M. B., The production of arterial hypertension by chronic renal artery-nerve stimulation. Am. J. Physiol., $1945-46,145,38$.

34. Goormaghtigh, N., Vascular and circulatory changes in the renal cortex in the anuric crush syndrome. Proc. Soc. Exper. Biol. \& Med., 1945, 59, 303.

35. Trueta, J., Barclay, A. E., Daniel, P. M., Franklin, K. J., and Prichard, M. L., Studies of the Renal Circulation. Charles C. Thomas, Springfield, Ill., 1947. 